\title{
Quantum field theoretical description of the Casimir effect between two real graphene sheets and thermodynamics
}

\author{
G. L. Klimchitskaya ${ }^{1,2}$ and V. M. Mostepanenko ${ }^{1,2,3}$ \\ ${ }^{1}$ Central Astronomical Observatory at Pulkovo of the Russian \\ Academy of Sciences, Saint Petersburg, 196140, Russia \\ ${ }^{2}$ Institute of Physics, Nanotechnology and Telecommunications, \\ Peter the Great Saint Petersburg Polytechnic University, Saint Petersburg, 195251, Russia \\ ${ }^{3}$ Kazan Federal University, Kazan, 420008, Russia
}

\begin{abstract}
The analytic asymptotic expressions for the Casimir free energy and entropy for two parallel graphene sheets possessing nonzero energy gap $\Delta$ and chemical potential $\mu$ are derived at arbitrarily low temperature. Graphene is described in the framework of thermal quantum field theory in the Matsubara formulation by means of the polarization tensor in $(2+1)$-dimensional space-time. Different asymptotic expressions are found under the conditions $\Delta>2 \mu, \Delta=2 \mu$, and $\Delta<$ $2 \mu$ taking into account both the implicit temperature dependence due to a summation over the Matsubara frequencies and the explicit one caused by a dependence of the polarization tensor on temperature as a parameter. It is shown that for both $\Delta>2 \mu$ and $\Delta<2 \mu$ the Casimir entropy satisfies the third law of thermodynamics (the Nernst heat theorem), whereas for $\Delta=2 \mu$ this fundamental requirement is violated. The physical meaning of the discovered anomaly is considered in the context of thermodynamic properties of the Casimir effect between metallic and dielectric bodies.
\end{abstract}




\section{INTRODUCTION}

The Casimir effect was discovered [1] as an attractive force which arises between two parallel uncharged ideal metal planes in vacuum and depends only on the Planck constant $\hbar$, speed of light $c$, and interplane distance $a$. At zero temperature of the planes this effect is entirely caused by the zero-point oscillations of the quantized electromagnetic field whose spectrum is altered by the presence of boundary conditions on the planes as compared to the free Minkowski space. More recently, the Casimir effect was generalized to the case of metallic or dielectric plates kept at arbitrary temperature $T$. In the framework of the Lifshitz theory, the free energy and force of the Casimir interaction between real-material plates are represented as some functionals of the reflection coefficients expressed via the frequency-dependent dielectric permittivities of plate materials. Detailed information on calculation of the Casimir free energies and forces using the Lifshitz theory, as well as about a comparison between experiment and theory, can be found in the monograph [2]. There are also generalizations of the Lifshitz theory for bodies of arbitrary shape and alternative derivations of the Casimir interaction in the literature (see, e.g., Refs. [2 -5]).

During the last few years, much attention is given to graphene which is a one-atomthick layer of carbon atoms possessing unusual physical properties [6]. It has been shown that at energies below 1-2 eV graphene is well described by the Dirac model as a set of massless or very light electronic quasiparticles. The corresponding fermion field satisfies the relativistic Dirac equation in $(2+1)$-dimensions where the speed of light $c$ is replaced with the Fermi velocity $v_{F} \approx c / 300[\underline{6}$, 7]. This allowed application of the methods developed earlier in planar quantum electrodynamics [8 11] for investigation of various quantum effects in graphene systems [12 18].

One of these effects is the Casimir attraction between two parallel graphene sheets which can be calculated using the Lifshitz theory [2]. For this purpose, one should know the response function of graphene to the electromagnetic field which does not reduce to the standard dielectric permittivities of metallic and dielectric materials. It is important to keep in mind that the permittivities of ordinary materials are usually derived using the kinetic theory or Kubo formula under several assumptions which are not universally applicable [19]. These ones and some other theoretical approaches have been used in approximate

calculations of the response functions and the Casimir force in graphene systems [20 37]. 
In the framework of the Dirac model, however, the dielectric response of graphene can be described exactly by means of its polarization tensor found on the basis of first principles of thermal quantum field theory.

Although the polarization tensor of graphene was considered in many papers (see, e.g., Ref. [38] and literature therein), the exact expression for it at zero temperature, as well as the corresponding formulas for the reflection coefficients, have been found in Ref. [39]. The polarization tensor of gapped graphene (the energy gap $\Delta$ arises for quasiparticles of nonzero mass) at any temperature was derived in Ref. [40]. The expressions of Ref. [40] are valid at the pure imaginary Matsubara frequencies and were used to investigate the Casimir effect in many graphene systems [40-50]. In Ref. [51] another form for the polarization tensor of graphene at nonzero temperature was derived valid over the entire plane of complex frequencies. It was generalized for the case of nonzero chemical potential $\mu$ in Ref. [52]. This form of the polarization tensor was also successfully used in calculations of the Casimir force in various graphene systems [52 -57] , as well as for investigation of the reflectivity and conductivity properties of graphene $\left[\begin{array}{lll}58 & 61\end{array}\right]$.

An interest to the thermodynamic aspects of the Lifshitz theory in application to graphene systems arose from the so-called Casimir puzzle. It turned out that the theoretical predictions for the Casimir force between both metallic and dielectric test bodies are excluded by the measurement data if one takes into account in calculations the dissipation of free electrons and the conductivity at a constant current, respectively (see the reviews in Refs. [2, 62, 63] and the most recent experiments $[64-67])$. As to thermodynamics, it was found that an account of dissipation of free electrons for metals with perfect crystal lattices and the dc conductivity for dielectrics results in a violation of the third law of thermodynamics which is also known as the Nernst heat theorem (see the reviews in Refs. [2, 62] and the most recent results in Refs. [68-73]). In the single experiment on measuring the Casimir interaction in graphene systems performed up to date [74], the measurement data were found in good agreement with theoretical predictions using the polarization tensor [75]. Taking into consideration that the polarization tensor of graphene results in two spatially nonlocal dielectric permittivities, the longitudinal one and the transverse one, each of which is complex and takes dissipation into account, the question arises whether the Casimir free energy and entropy of graphene systems is consistent with the requirements of thermodynamics.

To answer this question, the low-temperature behavior of the Casimir free energy and 
entropy between two sheets of pristine graphene with $\Delta=\mu=0$ was found in Ref. [76]. It was shown that in this case the Casimir entropy vanishes with vanishing temperature, i.e., the Nernst heat theorem is satisfied. The same result was obtained for the CasimirPolder entropy of an atom interacting with a sheet of a pristine graphene [77]. For an atom interacting with real graphene sheet possessing nonzero $\Delta$ and $\mu$ it was shown that the Nernst heat theorem is followed for $\Delta>2 \mu$ [78] and $\Delta<2 \mu$ [78, 79]. As to the case $\Delta=2 \mu$, the nonzero value of the Casimir-Polder entropy at zero temperature was found in this case depending on the parameters of a system, i.e., an entropic anomaly [79] (the low-temperature behavior of the Casimir-Polder free energy for $\Delta, \mu \neq 0$ was also considered in Ref. [80]).

In this paper, we derive the low-temperature analytic asymptotic expressions for the Casimir free energy and entropy of two real graphene sheets possessing nonzero values of $\Delta$ and $\mu$. This is a more complicated problem than for an atom interacting with real graphene sheet because the free energy of an atom-graphene interaction is the linear function of the reflection coefficients, which is not the case for two parallel graphene sheets. The Casimir free energy is presented by the Lifshitz formula where the reflection coefficients are expressed via the polarization tensor of graphene in $(2+1)$-dimensional space-time. The thermal correction to the Casimir energy at zero temperature is separated in two contributions. In the first of them, the temperature dependence is determined exclusively by a summation over the Matsubara frequencies, whereas the polarization tensor is defined at zero temperature. The temperature dependence of the second contribution is determined by an explicit dependence of the polarization tensor on temperature as a parameter.

We find the asymptotic behaviors at low temperature for each of these contributions under different relationships between $\Delta$ and $2 \mu$. It is shown that the leading terms determining the low-temperature behavior of the total Casimir free energy originate from the first contribution to the thermal correction for both $\Delta>2 \mu$ and $\Delta<2 \mu$ and from the second contribution for $\Delta=2 \mu$. As a result, for $\Delta>2 \mu$ and $\Delta<2 \mu$ the Nernst heat theorem is satisfied, whereas for $\Delta=2 \mu$ it is violated. The physical meaning of this anomaly is discussed in the context of problems considered earlier in the literature on the Casimir effect between metals and dielectrics.

The paper is organized as follows. In Sec. II, we briefly summarize the necessary formalism of the polarization tensor. Section III is devoted to the perturbation expansion of the Lifshitz 
formula at low temperature. In Secs. IV, V, and VI, the derivation of the asymptotic expressions for the Casimir free energy and entropy at low temperature is presented for the cases $\Delta>2 \mu, \Delta=2 \mu$, and $\Delta<2 \mu$, respectively. Section VII contains our conclusions and a discussion. In the Appendix, the reader will find some calculation details.

\section{THE POLARIZATION TENSOR OF GRAPHENE AND THE REFLECTION COEFFICIENTS}

We consider two parallel graphene sheets separated by a distance $a$ at temperature $T$ in thermal equilibrium with the environment. The electronic quasiparticles in graphene considered in the framework of the Dirac model [6, 7] are characterized by some small but nonzero mass which results in the energy gap $\Delta$ taking the typical value $0.1-0.2 \mathrm{eV}$. The energy gap arises due to an impact of the defects of structure, interelectron interactions and interaction with a substrate if any [38, 81]. We also assume that the graphene sheets under consideration possess some value of the chemical potential $\mu$ which depends on the doping concentration [82] (for a pristine graphene $\Delta=\mu=0$ ).

The polarization tensor of graphene describes its response to an external electromagnetic field in the one-loop approximation. The values of this tensor at the pure imaginary Matsubara frequencies $\xi_{l}=2 \pi k_{B} T l / \hbar$ (where $k_{B}$ is the Boltzmann constant and $l=0,1,2 \ldots$ ) are usually notated as

$$
\Pi_{m n}\left(\mathrm{i} \xi_{l}, k_{\perp}, T, \Delta, \mu\right) \equiv \Pi_{m n, l}\left(k_{\perp}, T, \Delta, \mu\right)
$$

where $m, n=0,1,2$ are the tensor indices and $k_{\perp}$ is the magnitude of the wave vector projection on the plane of graphene. Below it is convenient to consider the dimensionless polarization tensor, frequencies and the wave vector projection defined as

$$
\tilde{\Pi}_{m n . l}=\frac{2 a}{\hbar} \Pi_{m n, l}, \quad \zeta_{l}=\frac{\xi_{l}}{\omega_{c}}, \quad \omega_{c} \equiv \frac{c}{2 a}, \quad y=2 a\left(k_{\perp}^{2}+\frac{\xi_{l}^{2}}{c^{2}}\right)^{1 / 2} .
$$

In fact only the two components of the polarization tensor are the independent quantities. As an example, the 00 component $\tilde{\Pi}_{00}$ and the trace $\tilde{\Pi}_{m}^{m}$ are often used for a full characterization of this tensor [40]. For our purposes it is more convenient to use $\tilde{\Pi}_{00}$ and the following linear combination of the 00 component and the trace:

$$
\tilde{\Pi}_{l} \equiv \tilde{\Pi}\left(\mathrm{i} \zeta_{l}, y, T, \Delta, \mu\right)=\left(y^{2}-\zeta_{l}^{2}\right) \tilde{\Pi}_{m}^{m}\left(\mathrm{i} \zeta_{l}, y, T, \Delta, \mu\right)-y^{2} \tilde{\Pi}_{00}\left(\mathrm{i} \zeta_{l}, y, T, \Delta, \mu\right)
$$


The reason is that the reflection coefficients on graphene sheets for the transverse magnetic (TM) and transverse electric (TE) polarizations of the electromagnetic waves take the following simple form [39, 40, 51, 52]:

$$
\begin{aligned}
& r_{\mathrm{TM}}\left(\mathrm{i} \zeta_{l}, y, T\right)=\frac{y \tilde{\Pi}_{00, l}(y, T, \Delta, \mu)}{y \tilde{\Pi}_{00, l}(y, T, \Delta, \mu)+2\left(y^{2}-\zeta_{l}^{2}\right)}, \\
& r_{\mathrm{TE}}\left(\mathrm{i} \zeta_{l}, y, T\right)=-\frac{\tilde{\Pi}_{l}(y, T, \Delta, \mu)}{\tilde{\Pi}_{l}(y, T, \Delta, \mu)+2 y\left(y^{2}-\zeta_{l}^{2}\right)},
\end{aligned}
$$

where we omitted the parameters $\Delta$ and $\mu$ in the notations of the reflection coefficients for the sake of brevity.

Now we present the exact expressions for $\tilde{\Pi}_{00, l}$ and $\tilde{\Pi}_{l}$ obtained in the literature. First of all, it is convenient to present them as the respective quantity defined at $T=0$ plus the thermal correction to it

$$
\begin{aligned}
& \tilde{\Pi}_{00, l}(y, T, \Delta, \mu)=\tilde{\Pi}_{00, l}(y, 0, \Delta, \mu)+\delta_{T} \tilde{\Pi}_{00, l}(y, T, \Delta, \mu), \\
& \tilde{\Pi}_{l}(y, T, \Delta, \mu)=\tilde{\Pi}_{l}(y, 0, \Delta, \mu)+\delta_{T} \tilde{\Pi}_{l}(y, T, \Delta, \mu) .
\end{aligned}
$$

It is also useful to present $\tilde{\Pi}_{00, l}$ and $\tilde{\Pi}_{l}$ as the sums of contributions which do not depend and, quite the reverse, depend on $\mu$ and $T$ [57]

$$
\begin{aligned}
& \tilde{\Pi}_{00, l}(y, T, \Delta, \mu)=\tilde{\Pi}_{00, l}^{(0)}(y, \Delta)+\tilde{\Pi}_{00, l}^{(1)}(y, T, \Delta, \mu), \\
& \tilde{\Pi}_{l}(y, T, \Delta, \mu)=\tilde{\Pi}_{l}^{(0)}(y, \Delta)+\tilde{\Pi}_{l}^{(1)}(y, T, \Delta, \mu) .
\end{aligned}
$$

As the first contributions on the right-hand side of Eq. (6) we choose the 00 component and the combination (3) for the polarization tensor of gapped $(\Delta \neq 0)$ but undoped $(\mu=0)$ graphene defined at zero temperature [39, 57]

$$
\begin{aligned}
& \tilde{\Pi}_{00, l}^{(0)}(y, \Delta)=\alpha \frac{y^{2}-\zeta_{l}^{2}}{p_{l}} \Psi\left(\frac{D}{p_{l}}\right), \\
& \tilde{\Pi}_{l}^{(0)}(y, \Delta)=\alpha\left(y^{2}-\zeta_{l}^{2}\right) p_{l} \Psi\left(\frac{D}{p_{l}}\right),
\end{aligned}
$$

where $\alpha=e^{2} /(\hbar c)$ is the fine structure constant, $D \equiv \Delta /\left(\hbar \omega_{c}\right)$, and the following notations are introduced

$$
\Psi(x)=2\left[x+\left(1-x^{2}\right) \arctan \left(x^{-1}\right)\right], \quad p_{l}=\left[\tilde{v}_{F}^{2} y^{2}+\left(1-\tilde{v}_{F}^{2}\right) \zeta_{l}^{2}\right]^{1 / 2}, \quad \tilde{v}_{F}=\frac{v_{F}}{c} .
$$


In accordance to our choice,

$$
\begin{aligned}
& \tilde{\Pi}_{00, l}^{(0)}(y, \Delta)=\tilde{\Pi}_{00, l}(y, 0, \Delta, 0), \\
& \tilde{\Pi}_{l}^{(0)}(y, \Delta)=\tilde{\Pi}_{l}(y, 0, \Delta, 0) .
\end{aligned}
$$

In so doing, $\tilde{\Pi}_{00, l}^{(1)}$ and $\tilde{\Pi}_{l}^{(1)}$ acquire a meaning of the thermal corrections to the polarization tensor of undoped graphene defined at $T=0$ :

$$
\begin{aligned}
& \tilde{\Pi}_{00, l}^{(1)}(y, T, \Delta, 0)=\delta_{T} \tilde{\Pi}_{00, l}(y, T, \Delta, 0), \\
& \tilde{\Pi}_{l}^{(1)}(y, T, \Delta, 0)=\delta_{T} \tilde{\Pi}_{l}(y, T, \Delta, 0) .
\end{aligned}
$$

These corrections vanish in the limit of zero temperature.

The second contributions on the right-hand side of Eq. (6) can be explicitly presented in the form [57, 79]

$$
\begin{aligned}
& \tilde{\Pi}_{00, l}^{(1)}(y, T, \Delta, \mu)=\frac{4 \alpha D}{\tilde{v}_{F}^{2}} \int_{1}^{\infty} d t w(t, T, \Delta, \mu) X_{00, l}(t, y, D), \\
& \tilde{\Pi}_{l}^{(1)}(y, T, \Delta, \mu)=-\frac{4 \alpha D}{\tilde{v}_{F}^{2}} \int_{1}^{\infty} d t w(t, T, \Delta, \mu) X_{l}(t, y, D),
\end{aligned}
$$

where the $\mu$-dependent factor is given by

$$
w(t, T, \Delta, \mu)=\left(e^{\frac{t \Delta+2 \mu}{2 k_{B} T}}+1\right)^{-1}+\left(e^{\frac{t \Delta-2 \mu}{2 k_{B} T}}+1\right)^{-1}
$$

and the functions $X_{00, l}$ and $X_{l}$ are defined as follows:

$$
\begin{aligned}
& X_{00, l}(t, y, D)=1-\operatorname{Re} \frac{p_{l}^{2}-D^{2} t^{2}+2 \mathrm{i} \zeta_{l} D t}{\left[p_{l}^{4}-p_{l}^{2} D^{2} t^{2}+\tilde{v}_{F}^{2}\left(y^{2}-\zeta_{l}^{2}\right) D^{2}+2 \mathrm{i} \zeta_{l} p_{l}^{2} D t\right]^{1 / 2}}, \\
& X_{l}(t, y, D)=\zeta_{l}^{2}-\operatorname{Re} \frac{\zeta_{l}^{2} p_{l}^{2}-p_{l}^{2} D^{2} t^{2}+\tilde{v}_{F}^{2}\left(y^{2}-\zeta_{l}^{2}\right) D^{2}+2 \mathrm{i} \zeta_{l} p_{l}^{2} D t}{\left[p_{l}^{4}-p_{l}^{2} D^{2} t^{2}+\tilde{v}_{F}^{2}\left(y^{2}-\zeta_{l}^{2}\right) D^{2}+2 \mathrm{i} \zeta_{l} p_{l}^{2} D t\right]^{1 / 2}} .
\end{aligned}
$$

It has been shown [56, 57] that for a doped and gapped graphene satisfying the condition $\Delta \geqslant 2 \mu$ the polarization tensor at $T=0$ also does not depend on $\mu$. As a result, one obtains the equalities similar to those in Eqs. (9) and (10)

$$
\tilde{\Pi}_{00, l}(y, 0, \Delta, \mu)=\tilde{\Pi}_{00, l}^{(0)}(y, \Delta), \quad \tilde{\Pi}_{l}(y, 0, \Delta, \mu)=\tilde{\Pi}_{l}^{(0)}(y, \Delta),
$$

and

$$
\delta_{T} \tilde{\Pi}_{00, l}(y, T, \Delta, \mu)=\tilde{\Pi}_{00, l}^{(1)}(y, T, \Delta, \mu), \quad \delta_{T} \tilde{\Pi}_{l}(y, T, \Delta, \mu)=\tilde{\Pi}_{l}^{(1)}(y, T, \Delta, \mu),
$$


where the thermal corrections vanish with vanishing temperature.

It is significant that under the condition $\Delta<2 \mu$ the polarization tensor of doped and gapped graphene at $T=0$ depends both on $\Delta$ and $\mu$, and Eqs. (14) and (15) are not valid any more. In this case, the 00 component of the polarization tensor at $T=0$ and the combination of its components (3) are given by [56]

$$
\begin{gathered}
\tilde{\Pi}_{00, l}(y, 0, \Delta, \mu)=\frac{8 \alpha \mu}{\tilde{v}_{F}^{2} \hbar \omega_{c}}-\frac{2 \alpha\left(y^{2}-\zeta_{l}^{2}\right)}{p_{l}^{3}}\left\{\left(p_{l}^{2}+D^{2}\right) \operatorname{Im}\left(z_{l} \sqrt{1+z_{l}^{2}}\right)\right. \\
\left.+\left(p_{l}^{2}-D^{2}\right)\left[\operatorname{Im} \ln \left(z_{l}+\sqrt{1+z_{l}^{2}}\right)-\frac{\pi}{2}\right]\right\}, \\
\tilde{\Pi}_{l}(y, 0, \Delta, \mu)=-\frac{8 \alpha \mu \zeta_{l}^{2}}{\tilde{v}_{F}^{2} \hbar \omega_{c}}+\frac{2 \alpha\left(y^{2}-\zeta_{l}^{2}\right)}{p_{l}}\left\{\left(p_{l}^{2}+D^{2}\right) \operatorname{Im}\left(z_{l} \sqrt{1+z_{l}^{2}}\right)\right. \\
\left.-\left(p_{l}^{2}-D^{2}\right)\left[\operatorname{Im} \ln \left(z_{l}+\sqrt{1+z_{l}^{2}}\right)-\frac{\pi}{2}\right]\right\},
\end{gathered}
$$

where

$$
z_{l} \equiv z_{l}(y, \Delta, \mu)=\frac{p_{l}}{\tilde{v}_{F} \sqrt{p_{l}^{2}+D^{2}} \sqrt{y^{2}-\zeta_{l}^{2}}}\left(\zeta_{l}+\mathrm{i} \frac{2 \mu}{\hbar \omega_{c}}\right) .
$$

The thermal corrections to the polarization tensor of graphene satisfying the condition $\Delta<2 \mu$ are immediately obtained from Eqs. (5) and (6)

$$
\begin{aligned}
\delta_{T} \tilde{\Pi}_{00, l}(y, T, \Delta, \mu) & =\tilde{\Pi}_{00, l}(y, T, \Delta, \mu)-\tilde{\Pi}_{00, l}(y, 0, \Delta, \mu) \\
& =\tilde{\Pi}_{00, l}^{(1)}(y, T, \Delta, \mu)-\tilde{\Pi}_{00, l}^{(1)}(y, 0, \Delta, \mu), \\
\delta_{T} \tilde{\Pi}_{l}(y, T, \Delta, \mu)= & \tilde{\Pi}_{l}(y, T, \Delta, \mu)-\tilde{\Pi}_{l}(y, 0, \Delta, \mu) \\
= & \tilde{\Pi}_{l}^{(1)}(y, T, \Delta, \mu)-\tilde{\Pi}_{l}^{(1)}(y, 0, \Delta, \mu) .
\end{aligned}
$$

As to the case of an exact equality $\Delta=2 \mu$, it is considered in Sec. V.

\section{PERTURBATION EXPANSION OF THE LIFSHITZ FORMULA AT LOW TEMPERATURE}

Using the reflection coefficients (41) expressed above via the polarization tensor, one can represent the Casimir free energy per unit area of graphene sheets by means of the Lifshitz 
formula [2, 83]

$$
\mathcal{F}(a, T)=\frac{k_{B} T}{8 \pi a^{2}} \sum_{l=0}^{\infty} \int_{\zeta_{l}}^{\infty} y d y \sum_{\lambda} \ln \left[1-r_{\lambda}^{2}\left(\mathrm{i} \zeta_{l}, y, T\right) e^{-y}\right]
$$

where the prime on the summation sign divides the term with $l=0$ by 2 , and the sum in $\lambda$ is over two polarizations of the electromagnetic field, transverse magnetic and transverse electric $(\lambda=\mathrm{TM}, \mathrm{TE})$.

We are in fact interested not in the total Casimir free energy but in its temperaturedependent part, i.e., in the thermal correction to the Casimir energy defined as

$$
\delta_{T} \mathcal{F}(a, T)=\mathcal{F}(a, T)-E(a)
$$

where the Casimir energy at zero temperature is given by [2, 83]

$$
E(a)=\frac{\hbar c}{32 \pi^{2} a^{3}} \int_{0}^{\infty} d \zeta \int_{\zeta}^{\infty} y d y \sum_{\lambda} \ln \left[1-r_{\lambda}^{2}(\mathrm{i} \zeta, y, 0) e^{-y}\right]
$$

Here, the reflection coefficients are expressed by Eq. (4) in which one should replace the Matsubara frequencies with a continuous frequency $\zeta$ and put $T=0$

$$
\begin{aligned}
& r_{\mathrm{TM}}(\mathrm{i} \zeta, y, 0)=\frac{y \tilde{\Pi}_{00}(\mathrm{i} \zeta, y, 0, \Delta, \mu)}{y \tilde{\Pi}_{00}(\mathrm{i} \zeta, y, 0, \Delta, \mu)+2\left(y^{2}-\zeta^{2}\right)}, \\
& r_{\mathrm{TE}}(\mathrm{i} \zeta, y, 0)=-\frac{\tilde{\Pi}(\mathrm{i} \zeta, y, 0, \Delta, \mu)}{\tilde{\Pi}(\mathrm{i} \zeta, y, 0, \Delta, \mu)+2 y\left(y^{2}-\zeta^{2}\right)} .
\end{aligned}
$$

Note that both the propagating waves, which are on the mass shell, and the evanescent waves off the mass shell contribute to Eqs. (19) and (21).

In the case $\Delta>2 \mu$, following Eq. (14), one should substitute to Eq. (22) the expressions for the $\tilde{\Pi}_{00}$ and $\tilde{\Pi}$ defined in Eq. (7) making there the above replacement $\zeta_{l} \rightarrow \zeta$. If, however, the condition $\Delta<2 \mu$ is fulfilled, it is necessary to substitute in Eq. (22) the quantities (16) with the same replacement.

Now we identically rearrange Eq. (20) to the form

$$
\delta_{T} \mathcal{F}(a, T)=\delta_{T}^{\mathrm{impl}} \mathcal{F}(a, T)+\delta_{T}^{\text {expl }} \mathcal{F}(a, T)
$$

where 


$$
\delta_{T}^{\mathrm{impl}} \mathcal{F}(a, T)=\frac{k_{B} T}{8 \pi a^{2}} \sum_{l=0}^{\infty} \int_{\zeta_{l}}^{\infty} y d y \sum_{\lambda} \ln \left[1-r_{\lambda}^{2}\left(\mathrm{i} \zeta_{l}, y, 0\right) e^{-y}\right]-E(a)
$$

and

$$
\delta_{T}^{\operatorname{expl}} \mathcal{F}(a, T)=\mathcal{F}(a, T)-\frac{k_{B} T}{8 \pi a^{2}} \sum_{l=0}^{\infty} \int_{\zeta_{l}}^{\infty} y d y \sum_{\lambda} \ln \left[1-r_{\lambda}^{2}\left(\mathrm{i} \zeta_{l}, y, 0\right) e^{-y}\right] .
$$

As is seen from Eqs. (23)-(25), we have simply added and subtracted from Eq. (20) the quantity having the same form as the Casimir free energy in Eq. (19) but containing the reflection coefficients (4) taken at $T=0$.

An advantage of Eq. (23) is that the implicit temperature dependence of the first term, $\delta_{T}^{\mathrm{impl}} \mathcal{F}$, is entirely determined by a summation on the Matsubara frequencies, whereas the polarization tensor is taken at $T=0$. As to the second term, $\delta_{T}^{\operatorname{expl}} \mathcal{F}$, it simply vanishes for the temperature-independent polarization tensors. Thus, the dependence of this term on $T$ can be called explicit.

We turn our attention to the perturbation expansion of the Casimir free energy at low temperature. Taking into account that the thermal corrections $\delta_{T} \tilde{\Pi}_{00, l}$ and $\delta_{T} \tilde{\Pi}_{l}$ go to zero with vanishing $T$, we substitute Eq. (5) in Eq. (4), expand up to the first order of small parameters

$$
\frac{\delta_{T} \tilde{\Pi}_{00, l}(y, T, \Delta, \mu)}{\tilde{\Pi}_{00, l}(y, 0, \Delta, \mu)} \ll 1, \quad \frac{\delta_{T} \tilde{\Pi}_{l}(y, T, \Delta, \mu)}{\tilde{\Pi}_{l}(y, 0, \Delta, \mu)} \ll 1
$$

and obtain

$$
r_{\mathrm{TM}(\mathrm{TE})}\left(\mathrm{i} \zeta_{l}, y, T\right)=r_{\mathrm{TM}(\mathrm{TE})}\left(\mathrm{i} \zeta_{l}, y, 0\right)+\delta_{T} r_{\mathrm{TM}(\mathrm{TE})}\left(\mathrm{i} \zeta_{l}, y, T\right)
$$

where the first contributions are given by Eq. (41) taken at $T=0$ and the thermal corrections to the reflection coefficients are given by

$$
\begin{aligned}
& \delta_{T} r_{\mathrm{TM}}\left(\mathrm{i} \zeta_{l}, y, T\right)=\frac{2 y\left(y^{2}-\zeta_{l}^{2}\right) \delta_{T} \tilde{\Pi}_{00, l}(y, T, \Delta, \mu)}{\left[y \tilde{\Pi}_{00, l}(y, 0, \Delta, \mu)+2\left(y^{2}-\zeta_{l}^{2}\right)\right]^{2}}, \\
& \delta_{T} r_{\mathrm{TE}}\left(\mathrm{i} \zeta_{l}, y, T\right)=-\frac{2 y\left(y^{2}-\zeta_{l}^{2}\right) \delta_{T} \tilde{\Pi}_{l}(y, T, \Delta, \mu)}{\left[\tilde{\Pi}_{l}(y, 0, \Delta, \mu)+2 y\left(y^{2}-\zeta_{l}^{2}\right)\right]^{2}}
\end{aligned}
$$

This approach is applicable under the conditions $\tilde{\Pi}_{00, l}(y, 0, \Delta, \mu) \neq 0$ and $\tilde{\Pi}_{l}(y, 0, \Delta, \mu) \neq 0$ which are valid for the cases $\Delta \geqslant 2 \mu$ considered in Secs. IV and V. For the case $\Delta<2 \mu$, however, one cannot use the perturbation theory in the parameters (26) for the contribution of the Matsubara term with $l=0$ (see Sec. VI). 
The implicit thermal correction $\delta_{T}^{\text {impl }} \mathcal{F}$ defined in Eq. (24) is the difference between the sum in $l$ and the integral (21) with respect to $\zeta$. From Eq. (2) it is seen that $\zeta_{l}=\tau l$ where $\tau \equiv 4 \pi k_{B} T a /(\hbar c)$. By replacing the integration variable $\zeta$ in Eq. (21) with $t=\zeta / \tau$, one can bring Eq. (24) to the form

$$
\delta_{T}^{\mathrm{impl}} \mathcal{F}(a, T)=\frac{k_{B} T}{8 \pi a^{2}}\left[\sum_{l=0}^{\infty}{ }^{\prime} \Phi(\tau l)-\int_{0}^{\infty} d t \Phi(\tau t)\right]
$$

where

$$
\Phi(x)=\int_{x}^{\infty} y d y \sum_{\lambda_{\lambda}} \ln \left[1-r_{\lambda}^{2}(\mathrm{i} x, y, 0) e^{-y}\right] .
$$

By applying the Abel-Plana formula [2, 84], Eq. (29) can be rewritten as

$$
\delta_{T}^{\mathrm{impl}} \mathcal{F}(a, T)=\frac{\mathrm{i} k_{B} T}{8 \pi a^{2}} \int_{0}^{\infty} \frac{d t}{e^{2 \pi t}-1}[\Phi(\mathrm{i} \tau t)-\Phi(-\mathrm{i} \tau t)] .
$$

In the next sections, Eq. (31) is used to find the asymptotic behavior of $\delta_{T}^{\text {impl }} \mathcal{F}$ at arbitrarily low $T$.

In order to determine the low-temperature behavior of the second thermal correction to the Casimir energy, $\delta_{T}^{\text {expl }} \mathcal{F}$, we substitute Eq. (27) into its definition (25) and use the identity

$$
\begin{aligned}
& \ln \left\{1-\left[r_{\lambda}\left(\mathrm{i} \zeta_{l}, y, 0\right)+\delta_{T} r_{\lambda}\left(\mathrm{i} \zeta_{l}, y, T\right)\right]^{2} e^{-y}\right\}-\ln \left[1-r_{\lambda}^{2}\left(\mathrm{i} \zeta_{l}, y, 0\right) e^{-y}\right] \\
& =\ln \left\{1-\frac{2 r_{\lambda}\left(\mathrm{i} \zeta_{l}, y, 0\right) \delta_{T} r_{\lambda}\left(\mathrm{i} \zeta_{l}, y, T\right)+\left[\delta_{T} r_{\lambda}\left(\mathrm{i} \zeta_{l}, y, T\right)\right]^{2}}{1-r_{\lambda}^{2}\left(\mathrm{i} \zeta_{l}, y, 0\right) e^{-y}} e^{-y}\right\} .
\end{aligned}
$$

Then, expanding the logarithm up to the first power of a small parameter and preserving only the term of the first power in $\delta_{T} r_{\lambda}\left(\mathrm{i} \zeta_{l}, y, T\right)$, one arrives at

$$
\delta_{T}^{\operatorname{expl}} \mathcal{F}(a, T)=-\frac{k_{B} T}{4 \pi a^{2}} \sum_{l=0}^{\infty} \int_{\zeta_{l}}^{\infty} y d y e^{-y} \sum_{\lambda} \frac{r_{\lambda}\left(\mathrm{i} \zeta_{l}, y, 0\right) \delta_{T} r_{\lambda}\left(\mathrm{i} \zeta_{l}, y, T\right)}{1-r_{\lambda}^{2}\left(\mathrm{i} \zeta_{l}, y, 0\right) e^{-y}}
$$

This equation valid under a condition that $\tilde{\Pi}_{00, l}$ and $\tilde{\Pi}_{l}$ are nonzero at $T=0$ and, thus, $r_{\lambda}\left(\mathrm{i} \zeta_{l}, y, 0\right) \neq 0$ is used below to determine the behavior of $\delta_{T}^{\text {expl }} \mathcal{F}$ at low temperature.

\section{LOW-TEMPERATURE BEHAVIOR OF THE CASIMIR FREE ENERGY AND ENTROPY FOR GRAPHENE SHEETS WITH $\Delta>2 \mu$}

We assume that the graphene sheets under consideration in this section satisfy the con-

dition $\Delta>2 \mu$ and start with the thermal correction $\delta_{T}^{\text {impl }} \mathcal{F}(a, T)$ to the Casimir energy 
defined in Eq. (24) and expressed by Eqs. (29) and (31). In accordance to Eq. (30) the function $\Phi$ entering Eq. (29) is defined as the sum of contributions from the TM and TE modes

$$
\Phi(x)=\Phi_{\mathrm{TM}}(x)+\Phi_{\mathrm{TE}}(x) .
$$

As a result, $\delta_{T}^{\mathrm{impl}} \mathcal{F}(a, T)$ becomes the sum of $\delta_{T}^{\mathrm{impl}} \mathcal{F}_{\mathrm{TM}}(a, T)$ and $\delta_{T}^{\mathrm{impl}} \mathcal{F}_{\mathrm{TE}}(a, T)$.

Under the condition $\Delta>2 \mu$, the polarization tensor at $T=0$ is given by Eq. (17). By replacing $\zeta_{l}$ with $x$ in Eq. (17) and substituting the obtained expressions in Eq. (22) where $\zeta$ is also replaced with $x$, one obtains

$$
\begin{aligned}
& r_{\mathrm{TM}}(\mathrm{i} x, y, 0)=\frac{\alpha y \Psi\left(D p^{-1}\right)}{\alpha y \Psi\left(D p^{-1}\right)+2 p(x, y)}, \\
& r_{\mathrm{TE}}(\mathrm{i} x, y, 0)=-\frac{\alpha p(x, y) \Psi\left(D p^{-1}\right)}{\alpha p(x, y) \Psi\left(D p^{-1}\right)+2 y},
\end{aligned}
$$

where the quantity $p$ is defined as

$$
p \equiv p(x, y)=\left[\tilde{v}_{F}^{2} y^{2}+\left(1-\tilde{v}_{F}^{2}\right) x^{2}\right]^{1 / 2}
$$

In the analytic asymptotic expressions here and below we use the condition $\Delta>\hbar \omega_{c}$ (i.e., $D>1$ ) which is satisfied at not too small separations between the graphene sheets. Under this condition, at sufficiently small $x$ (low $T$ ) one can safely use the inequality $D \gg p(x, y$ ) because the dominant contribution to the integrals in Eq. (30) is given by $y \sim 1$.

We consider first the case $\lambda=$ TM. By expanding in Eq. (30) in Taylor series around $x_{0}=0$ with the help of the first formula in Eq. (35) and above condition, we find

$$
\begin{gathered}
\Phi_{\mathrm{TM}}(x)=\Phi_{\mathrm{TM}}(0)+\frac{4 \alpha^{2}}{9 D^{2}} x^{4}+\frac{16 \alpha^{2}(8 \alpha+3 D)}{135 D^{3}} x^{5}+O\left(x^{6}\right) \\
\approx \Phi_{\mathrm{TM}}(0)+\frac{4 \alpha^{2}}{9 D^{2}} x^{4}+\frac{16 \alpha^{2}}{45 D^{2}} x^{5}+O\left(x^{6}\right) .
\end{gathered}
$$

The first two terms on the right-hand side of this equation do not contribute to Eq. (31), whereas the third term leads to

$$
\Phi_{\mathrm{TM}}(\mathrm{i} \tau t)-\Phi_{\mathrm{TM}}(-\mathrm{i} \tau t)=\mathrm{i} \frac{32 \alpha^{2}}{45 D^{2}} \tau^{5} t^{5}
$$

Substituting this result in Eq. (31), one arrives at

$$
\delta_{T}^{\mathrm{impl}} \mathcal{F}_{\mathrm{TM}}(a, T)=-\frac{16 \alpha^{2} \pi^{4} a\left(k_{B} T\right)^{6}}{315 \Delta^{2}(\hbar c)^{3}} .
$$


We continue with the case $\lambda=\mathrm{TE}$. The function $\Phi_{\mathrm{TE}}(x)$ cannot be expanded in Taylor series around the point $x_{0}=0$. Because of this, we substitute the second line of Eq. (35) in Eq. (30), expand the integrand in powers of $x$ and integrate with respect to $y$ thereafter. The result is

$$
\begin{aligned}
\Phi_{\mathrm{TE}}(x) & =\left(\frac{4 \alpha}{3 D}\right)^{2}\left[-6 \tilde{v}_{F}^{4}-2 \tilde{v}_{F}^{2}\left(1-\tilde{v}_{F}^{2}\right) x^{2}+\tilde{v}_{F}^{2}\left(1-\frac{3}{4} \tilde{v}_{F}^{2}\right) x^{4}+\left(1-\tilde{v}_{F}^{2}\right) x^{4} \operatorname{Ei}(-x)\right. \\
& \left.-\frac{2 \tilde{v}_{F}^{2}}{3}\left(1-\frac{7}{10} \tilde{v}_{F}^{2}\right) x^{5}+O\left(x^{6}\right)\right],
\end{aligned}
$$

where $\operatorname{Ei}(z)$ in the exponential integral.

The first three terms on the right-hand side of this expression do not contribute to Eq. (31). The dominant contribution is given by the term containing the exponential integral which leads to

$$
\Phi_{\mathrm{TE}}(\mathrm{i} \tau t)-\Phi_{\mathrm{TE}}(-\mathrm{i} \tau t)=\mathrm{i} \pi\left(\frac{4 \alpha}{3 D}\right)^{2} \tau^{4} t^{4} .
$$

Substituting this equation in Eq. (31) and integrating, one arrives at the result

$$
\delta_{T}^{\mathrm{impl}} \mathcal{F}_{\mathrm{TE}}(a, T)=-\frac{32 \zeta(5) \alpha^{2}\left(k_{B} T\right)^{5}}{3 \pi^{2} \Delta^{2}(\hbar c)^{2}} .
$$

Comparing this with Eq. (39), we conclude that the dominant term in the asymptotic

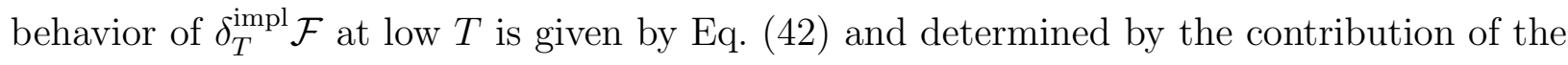
TE mode, i.e.,

$$
\delta_{T}^{\mathrm{impl}} \mathcal{F}(a, T)=\delta_{T}^{\mathrm{impl}} \mathcal{F}_{\mathrm{TE}}(a, T) \sim-\frac{\alpha^{2}\left(k_{B} T\right)^{5}}{\Delta^{2}(\hbar c)^{2}} .
$$

We are now coming to the asymptotic behavior of the second thermal correction, $\delta_{T}^{\operatorname{expl}} \mathcal{F}$, at low $T$ which takes into account an explicit dependence of the polarization tensor on temperature as a parameter. This correction is presented in Eq. (33). It is convenient to express $\delta_{T}^{\operatorname{expl}} \mathcal{F}$ as a sum of two contributions

$$
\delta_{T}^{\operatorname{expl}} \mathcal{F}(a, T)=\delta_{T, l=0}^{\operatorname{expl}} \mathcal{F}(a, T)+\delta_{T, l \geqslant 1}^{\operatorname{expl}} \mathcal{F}(a, T),
$$

where the first one contains the term of Eq. (33) with $l=0$ and the second one - all terms with $l \geqslant 1$.

We start from the first contribution on the right-hand side of Eq. (44). According to Eq. (33), it contains the zero-temperature reflection coefficients and thermal corrections to them, both taken at the zero Matsubara frequency. The reflection coefficients at $l=0$ are 
obtained from Eq. (35) by putting $x=0$

$$
\begin{aligned}
& r_{\mathrm{TM}}(0, y, 0)=\frac{\alpha \Psi\left(D \tilde{v}_{F}^{-1} y^{-1}\right)}{\alpha \Psi\left(D \tilde{v}_{F}^{-1} y^{-1}\right)+2 \tilde{v}_{F}}, \\
& r_{\mathrm{TE}}(0, y, 0)=-\frac{\alpha \tilde{v}_{F} \Psi\left(D \tilde{v}_{F}^{-1} y^{-1}\right)}{\alpha \tilde{v}_{F} \Psi\left(D \tilde{v}_{F}^{-1} y^{-1}\right)+2},
\end{aligned}
$$

Taking into account that for $y \sim 1$ it holds $\tilde{v}_{F} y \ll D$, we expand the function $\Psi$ in powers of the small parameter $\tilde{v}_{F} y / D$ and obtain

$$
\Psi\left(D \tilde{v}_{F}^{-1} y^{-1}\right) \approx \frac{8}{3} \frac{\tilde{v}_{F} y}{D}
$$

As a result, Eq. (45) reduces to

$$
\begin{aligned}
& r_{\mathrm{TM}}(0, y, 0) \approx \frac{\alpha y}{\alpha y+\frac{3}{4} D} \approx \frac{4 \alpha y}{3 D}, \\
& r_{\mathrm{TE}}(0, y, 0) \approx-\frac{\alpha \tilde{v}_{F}^{2} y}{\alpha \tilde{v}_{F}^{2} y+\frac{3}{4} D} \approx-\frac{4 \alpha \tilde{v}_{F}^{2} y}{3 D} .
\end{aligned}
$$

From Eq. (47) it is seen that

$$
r_{\mathrm{TE}}(0, y, 0) \approx-\tilde{v}_{F}^{2} r_{\mathrm{TM}}(0, y, 0)
$$

i.e., the magnitude of the TE reflection coefficient taken at zero frequency and temperature is negligibly small as compared to the TM one.

Next, we consider the thermal corrections to the reflection coefficients (47) entering Eq. (33). By putting $l=0$ in Eq. (28), one obtains

$$
\begin{aligned}
& \delta_{T} r_{\mathrm{TM}}(0, y, T)=\frac{2 y \delta_{T} \tilde{\Pi}_{00,0}(y, T, \Delta, \mu)}{\left[\tilde{\Pi}_{00,0}(y, 0, \Delta, \mu)+2 y\right]^{2}}, \\
& \delta_{T} r_{\mathrm{TE}}(0, y, T)=-\frac{2 y^{3} \delta_{T} \tilde{\Pi}_{0}(y, T, \Delta, \mu)}{\left[\tilde{\Pi}_{0}(y, 0, \Delta, \mu)+2 y^{3}\right]^{2}} .
\end{aligned}
$$

Under the condition $\Delta>2 \mu$ we can use Eq. (15) and, thus, the quantities $\delta_{T} \tilde{\Pi}_{00,0}$ and $\delta_{T} \tilde{\Pi}_{0}$ can be obtained from Eq. (11) taken at $l=0$. Taking into account that under the condition $\Delta>2 \mu$ the first contribution to Eq. (12) leads to an additional exponentially small factor $\exp \left[-2 \mu /\left(k_{B} T\right)\right]$, one can preserve only the second contribution. As a result, we have

$$
\delta_{T} \tilde{\Pi}_{00,0}(y, T, \Delta, \mu)=\frac{4 \alpha D}{\tilde{v}_{F}^{2}}\left[I_{00,0}^{(1)}+\frac{1}{\tilde{v}_{F} y} I_{00,0}^{(2)}\right] \text {, }
$$


where

$$
\begin{aligned}
& I_{00,0}^{(1)}=\int_{1}^{\infty} d t\left(e^{\frac{t \Delta-2 \mu}{2 k_{B} T}}+1\right)^{-1}, \\
& I_{00,0}^{(2)}=\int_{1}^{f(y, D)} d t\left(e^{\frac{t \Delta-2 \mu}{2 k_{B} T}}+1\right)^{-1} \frac{D^{2} t^{2}-\tilde{v}_{F}^{2} y^{2}}{\left[\tilde{v}_{F}^{2} y^{2}-D^{2}\left(t^{2}-1\right)\right]^{1 / 2}}
\end{aligned}
$$

and the function $f(y, D)$ is defined as

$$
f(y, D)=\sqrt{1+\frac{\tilde{v}_{F}^{2} y^{2}}{D^{2}}} .
$$

For the thermal correction $\delta_{T} \tilde{\Pi}_{0}$ from the second line in Eq. (11) one obtains

$$
\delta_{T} \tilde{\Pi}_{0}(y, T, \Delta, \mu)=-\frac{4 \alpha D^{3} y}{\tilde{v}_{F}} \int_{1}^{f(y, D)} d t\left(e^{\frac{t \Delta-2 \mu}{2 k_{B} T}}+1\right)^{-1} \frac{t^{2}-1}{\left[\tilde{v}_{F}^{2} y^{2}-D^{2}\left(t^{2}-1\right)\right]^{1 / 2}} .
$$

Since we consider arbitrarily low $T$, we can use the condition $\Delta-2 \mu \gg k_{B} T$. Under this condition the quantity $I_{00,0}^{(1)}$ in Eq. (51) takes an especially simple form

$$
I_{00,0}^{(1)} \approx \frac{2 k_{B} T}{\Delta} e^{-\frac{\Delta-2 \mu}{2 k_{B} T}}
$$

The quantity $I_{00,0}^{(2)}$ defined in Eq. (51) is calculated at low temperature in the Appendix. According to Eq. ( $(\underline{\mathrm{A} 3})$, the asymptotic behavior of $I_{00,0}^{(2)}$ is given by

$$
I_{00,0}^{(2)} \sim \frac{k_{B} T}{\tilde{v}_{F}} \frac{\Delta}{\left(\hbar \omega_{c}\right)^{2}} e^{-\frac{\Delta-2 \mu}{2 k_{B} T}}
$$

Then, from Eqs. (150), (154), and (55) we can conclude that

$$
\delta_{T} \tilde{\Pi}_{00,0}(y, T, \Delta, \mu) \sim \frac{\alpha k_{B} T}{\hbar \omega_{c}} e^{-\frac{\Delta-2 \mu}{2 k_{B} T}}\left(C_{1}+\frac{C_{2}}{y}\right),
$$

where $C_{1} \sim \tilde{v}_{F}^{-2}$ and $C_{2} \sim \tilde{v}_{F}^{-4}$ are the constants.

The integral with respect to $t$ in Eq. (53) for $\delta_{T} \tilde{\Pi}_{0}$ can be estimated similar to Eqs. (A2) and (A33). For this purpose, using Eq. (152), we replace $t^{2}-1$ with a larger quantity $\tilde{v}_{F}^{2} y^{2} / D^{2}$ and obtain

$$
\delta_{T} \tilde{\Pi}_{0}(y, T, \Delta, \mu) \sim-\frac{\alpha k_{B} T}{\hbar \omega_{c}} C_{3} e^{-\frac{\Delta-2 \mu}{2 k_{B} T}}
$$

where $C_{3} \sim \tilde{v}_{F}^{0}$.

Substituting Eqs. (7), (46), (56) and (57) in Eq. (49), one finds

$$
\begin{aligned}
& \delta_{T} r_{\mathrm{TM}}(0, y, T)=\frac{\delta_{T} \tilde{\Pi}_{00,0}(y, T, \Delta, \mu)}{2 y\left(\alpha y \frac{4}{3 D}+1\right)^{2}} \approx \frac{\delta_{T} \tilde{\Pi}_{00,0}(y, T, \Delta, \mu)}{2 y} \sim \frac{\alpha k_{B} T}{\hbar \omega_{c}} e^{-\frac{\Delta-2 \mu}{2 k_{B} T}}\left(\frac{C_{1}}{y}+\frac{C_{2}}{y^{2}}\right), \\
& \delta_{T} r_{\mathrm{TE}}(0, y, T)=-\frac{\delta_{T} \tilde{\Pi}_{0}(y, T, \Delta, \mu)}{2 y^{3}\left(\alpha \tilde{v}_{F}^{2} \frac{4 y}{3 D}+1\right)^{2}} \approx-\frac{\delta_{T} \tilde{\Pi}_{0}(y, T, \Delta, \mu)}{2 y^{3}} \sim \frac{\alpha k_{B} T}{\hbar \omega_{c} y^{3}} C_{3} e^{-\frac{\Delta-2 \mu}{2 k_{B} T}}
\end{aligned}
$$


From these equations, we obtain

$$
\delta_{T} r_{\mathrm{TE}}(0, y, T) \sim \tilde{v}_{F}^{4} \delta_{T} r_{\mathrm{TM}}(0, y, T)
$$

i.e., similar to Eq. (48), thermal correction to the TE reflection coefficient at zero Matsubara frequency is negligibly small comparing to the TM one.

Now we substitute the first lines of Eqs. (47) and (58) in the term of Eq. (33) with $l=0$ and obtain

$$
\delta_{T, l=0}^{\operatorname{expl}} \mathcal{F}(a, T) \approx \delta_{T, l=0}^{\operatorname{expl}} \mathcal{F}_{\mathrm{TM}}(a, T) \sim-\frac{\alpha^{2}\left(k_{B} T\right)^{2}}{a^{2} \Delta} e^{-\frac{\Delta-2 \mu}{2 k_{B} T}} \int_{0}^{\infty} d y e^{-y} \frac{C_{1} y+C_{2}}{1-\left(\frac{4 \alpha y}{3 D}\right)^{2} e^{-y}} .
$$

Taking into consideration that the integral in this equation converges, the final result is

$$
\delta_{T, l=0}^{\operatorname{expl}} \mathcal{F}(a, T) \sim-\frac{\alpha^{2}\left(k_{B} T\right)^{2}}{a^{2} \Delta} e^{-\frac{\Delta-2 \mu}{2 k_{B} T}}
$$

We are passing now to a consideration of the correction $\delta_{T, l \geqslant 1}^{\operatorname{expl}} \mathcal{F}$ which is equal to the sum of all terms with $l \geqslant 1$ in Eq. (33). In this case, from Eq. (35) with $x=\zeta_{l}$, using an approximate equality

$$
\Psi\left(\frac{D}{p_{l}}\right) \approx \frac{8}{3} \frac{p_{l}}{D}
$$

similar to Eq. (46), we find

$$
\begin{aligned}
& r_{\mathrm{TM}}\left(\mathrm{i} \zeta_{l}, y, 0\right) \approx \frac{\alpha y}{\alpha y+\frac{3}{4} D} \approx \frac{4 \alpha y}{3 D}, \\
& r_{\mathrm{TE}}\left(\mathrm{i} \zeta_{l}, y, 0\right) \approx-\frac{\alpha p_{l}^{2}}{\alpha p_{l}^{2}+\frac{3}{4} D y} \approx-\frac{4 \alpha p_{l}^{2}}{3 D y} \approx-\frac{4 \alpha \tilde{v}_{F}^{2} y}{3 D} .
\end{aligned}
$$

Here we have used that for $y \sim 1$, giving the dominant contribution to Eq. (33), $D \gg \alpha y$ and considered $p_{l} \approx \tilde{v}_{F} y$ at $\tau \rightarrow 0$. From Eq. (63) it is seen that similar to Eq. (48) relationship

$$
r_{\mathrm{TE}}\left(\mathrm{i} \zeta_{l}, y, 0\right) \approx-\tilde{v}_{F}^{2} r_{\mathrm{TM}}\left(\mathrm{i} \zeta_{l}, y, 0\right),
$$

holds at any $\zeta_{l}$.

Using Eq. (28), in the same approximation as in Eq. (58) one obtains

$$
\begin{aligned}
& \delta_{T} r_{\mathrm{TM}}\left(\mathrm{i} \zeta_{l}, y, T\right) \approx \frac{y \delta_{T} \tilde{\Pi}_{00, l}(y, T, \Delta, \mu)}{2\left(y^{2}-\zeta_{l}^{2}\right)}, \\
& \delta_{T} r_{\mathrm{TE}}\left(\mathrm{i} \zeta_{l}, y, T\right) \approx-\frac{\delta_{T} \tilde{\Pi}_{l}(y, T, \Delta, \mu)}{2 y\left(y^{2}-\zeta_{l}^{2}\right)} .
\end{aligned}
$$


From Eqs. (11), (13) and (15) one can make sure that

$$
\left.\delta_{T} \tilde{\Pi}_{00, l}(y, T, \Delta, \mu)\right|_{y=\zeta_{l}}=\left.\delta_{T} \tilde{\Pi}_{l}(y, T, \Delta, \mu)\right|_{y=\zeta_{l}}=0 .
$$

Because of this, the integrals with respect to $y$ in Eq. (33) are convergent at the low integration limit for all $l \geqslant 1$. Since the dominant contribution in Eq. (33) is given by $y \sim 1$, in the limiting case $\tau \rightarrow 0$ one can expand the integrand in Taylor series in the powers of $\zeta_{l}=\tau l$. For the order of magnitude estimation of the asymptotic behavior at $T \rightarrow 0$, it will suffice to consider the lowest expansion order. In this way, from Eqs. (333), (56) and (65) we find

$$
\begin{gathered}
\delta_{T, l \geqslant 1}^{\operatorname{expl}} \mathcal{F}_{\mathrm{TM}}(a, T) \sim-\frac{k_{B} T}{a^{2}} \sum_{l=1}^{\infty} \int_{\zeta_{l}}^{\infty} y d y e^{-y} \frac{r_{\mathrm{TM}}(0, y, 0)}{1-r_{\mathrm{TM}}^{2}(0, y, 0) e^{-y}} \frac{\delta_{T} \tilde{\Pi}_{00,0}(y, T, \Delta, \mu)}{y} \\
\sim-\frac{\alpha\left(k_{B} T\right)^{2}}{\hbar c a} e^{-\frac{\Delta-2 \mu}{2 k_{B} T}} \sum_{l=1}^{\infty} \int_{\zeta_{l}}^{\infty} d y e^{-y} \frac{r_{\mathrm{TM}}(0, y, 0)}{1-r_{\mathrm{TM}}^{2}(0, y, 0) e^{-y}}\left(C_{1}+\frac{C_{2}}{y}\right) .
\end{gathered}
$$

By introducing the variable $v=y / \zeta_{l}$ and using Eq. (63), it is seen that in the asymptotic limit $\tau \rightarrow 0$ the denominator in Eq. (67) can be replaced with unity and, thus,

$$
\begin{aligned}
& \delta_{T, l \geqslant 1}^{\operatorname{expl}} \mathcal{F}_{\mathrm{TM}}(a, T) \sim-\frac{\alpha^{2}\left(k_{B} T\right)^{2}}{\hbar c a} e^{-\frac{\Delta-2 \mu}{2 k_{B} T}} \sum_{l=1}^{\infty} \zeta_{l}^{2} \int_{\zeta_{l}}^{\infty} v d v e^{-\zeta_{l} v}\left(C_{1}+\frac{C_{2}}{\zeta_{l} v}\right) \\
& =-\frac{\alpha^{2}\left(k_{B} T\right)^{2}}{\hbar c a} e^{-\frac{\Delta-2 \mu}{2 k_{B} T}} \sum_{l=1}^{\infty}\left[C_{1}\left(1+\zeta_{l}\right)+C_{2}\right] e^{-\zeta_{l}} \sim-\frac{\alpha^{2}\left(k_{B} T\right)^{2}}{\hbar c a} e^{-\frac{\Delta-2 \mu}{2 k_{B} T}} \frac{1}{\tau} \sim-\frac{\alpha^{2} k_{B} T}{a^{2}} e^{-\frac{\Delta-2 \mu}{2 k_{B} T}} .
\end{aligned}
$$

Similar estimation shows that the contribution of the TE mode to Eq. (33) is again negligibly small

$$
\delta_{T, l \geqslant 1}^{\operatorname{expl}} \mathcal{F}_{\mathrm{TE}}(a, T) \sim \tilde{v}_{F}^{2} \delta_{T, l \geqslant 1}^{\mathrm{expl}} \mathcal{F}_{\mathrm{TM}}(a, T) .
$$

Because of this, the result is

$$
\delta_{T, l \geqslant 1}^{\operatorname{expl}} \mathcal{F}(a, T) \sim \delta_{T, l \geqslant 1}^{\operatorname{expl}} \mathcal{F}_{\mathrm{TM}}(a, T) \sim-\frac{\alpha^{2} k_{B} T}{a^{2}} e^{-\frac{\Delta-2 \mu}{2 k_{B} T}}
$$

Comparing Eqs. (61) and (70), we notice that a summation over the nonzero Matsubara frequencies decreases by one the power of temperature in front of the main exponential factor. Note also that Eqs. (43), (161), and (170) are obtained under the condition $\Delta>\hbar \omega_{c}$ and, thus, one cannot put there $\Delta=0$. These equations, however, are well applicable for graphene with $\mu=0$. 
Now we can find the dominant asymptotic behavior of the total thermal correction to the Casimir energy at zero temperature $\delta_{T} \mathcal{F}$ in the limit of low temperature. Taking into account that in accordance to Eqs. (23)) and (44) $\delta_{T} \mathcal{F}$ is given by the sum of Eqs. (43), (61), and (70), one concludes that under a condition $\Delta>2 \mu$ its leading behavior is given by Eq. (43), i.e.,

$$
\delta_{T} \mathcal{F}(a, T) \sim-\frac{\alpha^{2}\left(k_{B} T\right)^{5}}{\Delta^{2}(\hbar c)^{2}},
$$

and is determined by the TE contribution to the implicit temperature dependence.

This result gives the possibility to find the low-temperature behavior of the Casimir entropy per unit area of the graphene sheets defined as

$$
S(a, T)=-\frac{\partial \mathcal{F}(a, T)}{\partial T}=-\frac{\partial \delta_{T} \mathcal{F}(a, T)}{\partial T} .
$$

Using Eq. (71), one finds

$$
S(a, T) \sim \frac{\alpha^{2} k_{B}^{5} T^{4}}{\Delta^{2}(\hbar c)^{2}},
$$

which vanishes with vanishing temperature in agreement with the third law of thermodynamics (the Nernst heat theorem) [85, 86]. This means that the Lifshitz theory using the response function of graphene with $\Delta>2 \mu$ expressed in terms of the polarization tensor is thermodynamically consistent.

To summarize the application region of the obtained results, in this section we used the conditions

$$
k_{B} T \ll \frac{\hbar v_{F}}{2 a} \ll \frac{\hbar c}{2 a}<\Delta, \quad k_{B} T \ll \Delta-2 \mu
$$

and made the asymptotic expansions in three small parameters

$$
\tau \equiv \frac{4 \pi k_{B} T a}{\hbar c} \ll 1, \quad \frac{\hbar v_{F}}{2 a \Delta} \ll 1, \quad e^{-\frac{\Delta-2 \mu}{2 k_{B} T}} \ll 1 .
$$

The last parameter was used in finding the low-temperature behavior of $\delta_{T}^{\operatorname{expl}} \mathcal{F}$. It is possible, however, to dispense with this parameter (see the next section).

\section{LOW-TEMPERATURE BEHAVIOR OF THE CASIMIR FREE ENERGY AND ENTROPY FOR GRAPHENE SHEETS WITH $\Delta=2 \mu$}

As was stated in Sec. II, Eqs. (14) and (15) preserve their validity in the case $\Delta=2 \mu$. Because of this, all the results for $\delta_{T}^{\text {impl }} \mathcal{F}$ obtained in Sec. III for the graphene sheets with 
$\Delta>2 \mu$ remain valid in the case $\Delta=2 \mu$. Specifically, the low-temperature behavior of $\delta_{T}^{\mathrm{impl}} \mathcal{F}$ is again determined by the TE mode and is given by Eq. (43).

An explicit temperature dependence, however, leads to a radically different results. Although Eqs. (44)-(53) remain valid in the case $\Delta=2 \mu$, the subsequent equations obtained under a condition $\Delta-2 \mu \gg k_{B} T$ are not applicable. Thus, instead of Eq. (54), from the first line of Eq. (51) we obtain

$$
I_{00,0}^{(1)}=\frac{2 k_{B} T}{\Delta} \ln 2 .
$$

A more exact calculation of the integral $I_{00,0}^{(2)}$ defined in Eqs. (51) and (52) in the case $\Delta=2 \mu$ (see Appendix) in accordance to Eq. (A6) results in

$$
I_{00,0}^{(2)} \sim \frac{k_{B} T}{\tilde{v}_{F}} \frac{\Delta}{\left(\hbar \omega_{c}\right)^{2}} \ln 2 .
$$

As is seen from the comparison of Eqs. (76) and (77) with Eqs. (54) and (55), respectively, the values of $I_{00,0}^{(1)}$ and $I_{00,0}^{(2)}$ in the cases $\Delta>2 \mu$ and $\Delta=2 \mu$ differ only by the missing exponential factor and by an occurrence of the factor $\ln 2$ in the latter case. This allows to conclude that, similar to the case $\Delta>2 \mu$ considered in Sec. IV, the dominant contribution to the thermal correction $\delta_{T, l=0}^{\text {expl }} \mathcal{F}$ is determined by the TM mode. Up to an order of magnitude estimation of this contribution for the case $\Delta=2 \mu$, in accordance to Eq. (61), is given by

$$
\delta_{T, l=0}^{\operatorname{expl}} \mathcal{F}(a, T) \sim-\frac{\alpha^{2}\left(k_{B} T\right)^{2}}{a^{2} \Delta} .
$$

In a similar way, by repeating the derivation in Eqs. (62)-(701), one arrives at a conclusion that for $\Delta=2 \mu$ the contribution $\delta_{T, l \geqslant 1}^{\operatorname{expl}} \mathcal{F}$ to the thermal correction at low temperature is estimated by Eq. (70) where the exponential factor is replaced with unity

$$
\delta_{T, l \geqslant 1}^{\operatorname{expl}} \mathcal{F}(a, T) \sim-\frac{\alpha^{2} k_{B} T}{a^{2}} .
$$

From the comparison of Eq. (43) for an implicit contribution to the thermal correction, which is valid also for the case $\Delta=2 \mu$, with the explicit contributions (78) and (79), one concludes that in this case the low-temperature behavior of the total thermal correction is given by

$$
\delta_{T} \mathcal{F}(a, T) \sim-\frac{\alpha^{2} k_{B} T}{a^{2}},
$$

which originates from the TM mode in an explicit temperature dependence. In the case $\Delta=2 \mu$, Eqs. (43) and (78) -(80) are obtained under the first set of inequalities in Eq. (744), 
i.e., do not using the condition $k_{B} T \ll \Delta-2 \mu$. They employ only the first two small parameters indicated in Eq. (75) and are valid for graphene with $\Delta \neq 0$ and $\mu \neq 0$.

The result (80) leads to problems. The point is that, in accordance to Eq. (72)), the respective Casimir entropy per unit area of the graphene sheets at low temperature behaves as

$$
S(a, T) \sim \frac{\alpha^{2} k_{B}}{a^{2}} .
$$

Thus, the Casimir entropy at zero temperature is the nonzero (positive) constant depending on the volume of a system in violation of the Nernst heat theorem [85, 86]. As discussed in Sec. I, the same situation holds for metals with perfect crystal lattices described by the dielectric permittivity of the Drude model which, as opposed to the polarization tensor of graphene, is not derived from the first principles of quantum field theory. It should be taken into consideration, however, that for a real graphene sheet the values of $\Delta$ and $\mu$ cannot be known precisely. Thus, from the practical standpoint, the equality $\Delta=2 \mu$ can be considered as some singular point (see further discussion in Sec. VII). It is only important what are the properties of the Casimir free energy and entropy at low temperatures for graphene sheets with $\Delta<2 \mu$. This question is answered in the next section.

\section{LOW-TEMPERATURE BEHAVIOR OF THE CASIMIR FREE ENERGY AND ENTROPY FOR GRAPHENE SHEETS WITH $\Delta<2 \mu$}

Here, we consider the last possibility when the chemical potential is relatively large by exceeding the half of the energy gap. As in two preceding sections, we begin with consideration of the implicit contribution to the thermal correction given by Eq. (31), where the function $\Phi(x)$ is expressed via the reflection coefficients at zero temperature by Eq. (30).

In order to find these reflection coefficients, we consider the polarization tensor (16) and (17) found in the case $\Delta<2 \mu$, replace $\zeta_{l}$ with $x$ in Eqs. (16) and (17) and expand the results up to the first power in $x$ under the condition $\sqrt{4 \mu^{2}-\Delta^{2}}>\hbar \omega_{c}$ which is satisfied at not too small separations between the graphene sheets. The result is

$$
\tilde{\Pi}_{00}(x, y, 0, \Delta, \mu)=Q_{0}-Q_{1} \frac{x}{y}, \quad \tilde{\Pi}(x, y, 0, \Delta, \mu)=Q_{2} y x,
$$

where the following notations are introduced

$$
Q_{0}=\frac{4 \alpha}{\tilde{v}_{F}^{2}} \frac{2 \mu}{\hbar \omega_{c}}, \quad Q_{1}=\frac{16 \alpha \mu^{2}}{\tilde{v}_{F}^{3} \hbar \omega_{c} \sqrt{4 \mu^{2}-\Delta^{2}}}, \quad Q_{2}=\frac{4 \alpha \sqrt{4 \mu^{2}-\Delta^{2}}}{\tilde{v}_{F} \hbar \omega_{c}} .
$$


It is easily seen that under the used conditions $Q_{0} \gg 1$ holds.

We consider first the TM contribution to the function $\Phi(x)$ in Eqs. (301) and (34) and expand it up to the first power in small $x$

$$
\Phi_{\mathrm{TM}}(x)=\Phi_{\mathrm{TM}}(0)+x \Phi_{\mathrm{TM}}^{\prime}(0) .
$$

Substituting Eq. (82) in the first line of Eq. (22), where $\zeta$ is replaced with $x$, one obtains

$$
\begin{aligned}
& r_{\mathrm{TM}}(x, y, 0)=\frac{y Q_{0}-Q_{1} x}{y Q_{0}-Q_{1} x+2\left(y^{2}-x^{2}\right)}, \\
& r_{\mathrm{TM}}(0, y, 0)=\frac{Q_{0}}{Q_{0}+2 y} .
\end{aligned}
$$

From Eq. (30) at $\lambda=$ TM, using Eq. (85), it is easily seen that the quantity $\Phi_{\mathrm{TM}}(x)$ at $x=0$ is represented by a converging integral. Calculating the first derivative of $\Phi_{\mathrm{TM}}(x)$, one obtains

$$
\Phi_{\mathrm{TM}}^{\prime}(x)=-x \ln \left(1-e^{-x}\right)-\int_{x}^{\infty} y d y \frac{2 r_{\mathrm{TM}}(x, y, 0) e^{-y}}{1-r_{\mathrm{TM}}^{2}(x, y, 0) e^{-y}} \frac{\partial r_{\mathrm{TM}}(x, y, 0)}{\partial x} .
$$

By differentiating the first equality in Eq. (85), one finds

$$
\left.\frac{\partial r_{\mathrm{TM}}(x, y, 0)}{\partial x}\right|_{x=0}=-\frac{2 Q_{1}}{\left(Q_{0}+2 y\right)^{2}} .
$$

Then, substituting Eq. (87) in Eq. (86), we have

$$
\Phi_{\mathrm{TM}}^{\prime}(0)=4 Q_{1} \int_{0}^{\infty} d y \frac{y}{\left(Q_{0}+2 y\right)^{2}} \frac{r_{\mathrm{TM}}(0, y, 0) e^{-y}}{1-r_{\mathrm{TM}}^{2}(0, y, 0) e^{-y}} .
$$

Taking into account that $Q_{0} \gg 1$ and that the main contribution to the integral is given by $y \sim 1$, one finds from the second equality in Eq. (85) that $r_{\mathrm{TM}}(0, y, 0) \approx 1$. In such a manner, Eq. (88) reduces to

$$
\Phi_{\mathrm{TM}}^{\prime}(0) \approx \frac{4 Q_{1}}{Q_{0}^{2}} \int_{0}^{\infty} \frac{y d y}{e^{y}-1}=\frac{2 \pi^{2} Q_{1}}{3 Q_{0}^{2}} .
$$

Substituting this equation in Eq. (84), one obtains

$$
\Phi_{\mathrm{TM}}(\mathrm{i} \tau t)-\Phi_{\mathrm{TM}}(-\mathrm{i} \tau t)=\mathrm{i} \frac{4 \pi^{2} Q_{1}}{3 Q_{0}^{2}} \tau T .
$$

Now we consider the contribution of the TE mode in Eqs. (30) and (34). In this case the reflection coefficient is obtained by substituting Eq. (82) in the second line of Eq. (22)

$$
r_{\mathrm{TE}}(x, y, 0)=-\frac{Q_{2} x}{Q_{2} x+2\left(y^{2}-x^{2}\right)} .
$$


As is seen from this equation, $r_{\mathrm{TE}}(x, y, 0)$ goes to zero with vanishing $x$.

Using the first expansion term in the powers of $r_{\mathrm{TE}}(x, y, 0)$ in Eq. (30), we find

$$
\Phi_{\mathrm{TE}}(x) \approx-\int_{x}^{\infty} y d y r_{\mathrm{TE}}^{2}(x, y, 0) e^{-y} .
$$

Substituting here Eq. (91), one obtains

$$
\begin{aligned}
& \Phi_{\mathrm{TE}}(x) \approx-Q_{2}^{2} x^{2} \int_{x}^{\infty} d y \frac{y e^{-y}}{\left[Q_{2} x+2\left(y^{2}-x^{2}\right)\right]^{2}} \\
& \approx-\frac{Q_{2}^{2} x^{2}}{4} \int_{x}^{\infty} d y \frac{e^{-y}}{y^{3}}=\frac{Q_{2}^{2} x^{2}}{8}\left[\operatorname{Ei}(-x)-\frac{e^{-x}(1-x)}{x^{2}}\right] \\
& \approx-\frac{1}{8} Q_{2}^{2}\left[1-2 x+x^{2} \ln x+O\left(x^{2}\right)\right] .
\end{aligned}
$$

From this equation, the difference of our interest is given by

$$
\Phi_{\mathrm{TE}}(\mathrm{i} \tau t)-\Phi_{\mathrm{TE}}(-\mathrm{i} \tau t)=\mathrm{i} \frac{Q_{2}^{2}}{2} \tau t
$$

Comparing the difference in Eq. (90) with that in Eq. (94), one finds that the latter is larger than the former by the factor

$$
\frac{3 Q_{0}^{2} Q_{2}^{2}}{8 \pi^{2} Q_{1}}=\frac{24}{\pi^{2}}\left(\frac{\alpha \sqrt{4 \mu^{2}-\Delta^{2}}}{\tilde{v}_{F} \hbar \omega_{c}}\right)^{3} \gg 1 .
$$

Thus, one can approximately put

$$
\Phi(\mathrm{i} \tau t)-\Phi(-\mathrm{i} \tau t) \approx \Phi_{\mathrm{TE}}(\mathrm{i} \tau t)-\Phi_{\mathrm{TE}}(-\mathrm{i} \tau t) .
$$

Finally, substituting Eqs. (94) and (96) in Eq. (31), one arrives at the result

$$
\delta_{T}^{\mathrm{impl}} \mathcal{F}(a, T) \approx-\frac{k_{B} T}{16 \pi a^{2}} Q_{2}^{2} \tau \int_{0}^{\infty} \frac{t d t}{e^{2 \pi t}-1}=-\frac{4 \alpha^{2} a\left(k_{B} T\right)^{2}\left(4 \mu^{2}-\Delta^{2}\right)}{3 \tilde{v}_{F}^{2}(\hbar c)^{3}} .
$$

This result is obtained under a condition $\sqrt{4 \mu^{2}-\Delta^{2}}>\hbar \omega_{c}$ and, thus, $\mu \neq 0$. However, $\Delta=0$ is allowed.

Now we consider the explicit contributions to the thermal correction in the case $\Delta<2 \mu$ starting with $\delta_{T, l=0}^{\operatorname{expl}} \mathcal{F}$. We again use the condition $\sqrt{4 \mu^{2}-\Delta^{2}}>\hbar \omega_{c}$. Under this condition, in accordance with Eq. (82), $\tilde{\Pi}_{00,0}(y, 0, \Delta, \mu)=Q_{0} \neq 0$ and the reflection coefficient $r_{\mathrm{TM}}(0, y, 0)$ is given by the second expression in Eq. (85) and, thus, is not equal to zero. Because of this, for calculating the TM contribution to $\delta_{T, l=0}^{\text {expl }} \mathcal{F}$ one can use the term with $l=0$ in Eq. (33). 
The TE contribution to $\delta_{T, l=0}^{\operatorname{expl}} \mathcal{F}$ is a different matter. Here, in accordance to the second formula in Eq. (82), $\tilde{\Pi}_{0}(y, 0, \Delta, \mu)=0$ and, due to Eq. (91), $r_{\mathrm{TE}}(0, y, 0)=0$. Because of this, Eq. (33) is not applicable in this case and one should calculate $\delta_{T, l=0}^{\operatorname{expl}} \mathcal{F}_{\mathrm{TE}}$ using its definition as the term with $l=0$ in Eq. (25). Taking into account that due to the equality $r_{\mathrm{TE}}(0, y, 0)=0$ one has $r_{\mathrm{TE}}(0, y, T)=\delta_{T} r_{\mathrm{TE}}(0, y, T)$, Eq. (25) leads to

$$
\begin{aligned}
& \delta_{T, l=0}^{\operatorname{expl}} \mathcal{F}_{\mathrm{TE}}(a, T)=\frac{k_{B} T}{16 \pi a^{2}} \int_{0}^{\infty} y d y \ln \left\{1-\left[\delta_{T} r_{\mathrm{TE}}(0, y, T)\right]^{2} e^{-y}\right\} \\
& \approx-\frac{k_{B} T}{16 \pi a^{2}} \int_{0}^{\infty} y d y\left[\delta_{T} r_{\mathrm{TE}}(0, y, T)\right]^{2} e^{-y}
\end{aligned}
$$

where the last transformation is valid at sufficiently low $T$.

The thermal correction to the TM reflection coefficient in Eq. (33), in accordance to Eqs. (49) and (82) taken at $x=0$, is given by

$$
\delta_{T} r_{\mathrm{TM}}(0, y, T)=\frac{2 y \delta_{T} \tilde{\Pi}_{00,0}(y, T, \Delta, \mu)}{\left(Q_{0}+2 y\right)^{2}} .
$$

For obtaining $\delta_{T} r_{\mathrm{TE}}$, Eq. (49) is not applicable, so that it is found using Eq. (4) taken at $l=0$ with account of the equalities $\tilde{\Pi}_{0}=\delta_{T} \tilde{\Pi}_{0}$ and $r_{\mathrm{TE}}(0, y, T)=\delta_{T} r_{\mathrm{TE}}(0, y, T)$

$$
\delta_{T} r_{\mathrm{TE}}(0, y, T)=-\frac{\delta_{T} \tilde{\Pi}_{0}(y, T, \Delta, \mu)}{\delta_{T} \tilde{\Pi}_{0}(y, T, \Delta, \mu)+2 y^{3}} \approx-\frac{\delta_{T} \tilde{\Pi}_{0}(y, T, \Delta, \mu)}{2 y^{3}} .
$$

In the last transformation we have taken into account that the dominant contribution to Eq. (98) is given by $y \sim 1$ and that $\delta_{T} \tilde{\Pi}_{0}$ goes to zero with vanishing $T$.

In the case $\Delta<2 \mu$ under consideration now, the quantities $\delta_{T} \tilde{\Pi}_{00,0}$ and $\delta_{T} \tilde{\Pi}_{0}$, entering Eqs. (99) and (100), can be found from Eqs. (11) and (18)

$$
\begin{aligned}
& \delta_{T} \tilde{\Pi}_{00,0}(y, T, \Delta, \mu)=\frac{4 \alpha D}{\tilde{v}_{F}^{2}}\left[\int_{1}^{\infty} d t\left(e^{\frac{t \Delta-2 \mu}{2 k_{B} T}}+1\right)^{-1} X_{00,0}(t, y, D)-\int_{1}^{2 \mu / \Delta} d t X_{00,0}(t, y, D)\right] \\
& \delta_{T} \tilde{\Pi}_{0}(y, T, \Delta, \mu)=\frac{4 \alpha D}{\tilde{v}_{F}^{2}}\left[\int_{1}^{\infty} d t\left(e^{\frac{t \Delta-2 \mu}{2 k_{B} T}}+1\right)^{-1} X_{0}(t, y, D)-\int_{1}^{2 \mu / \Delta} d t X_{0}(t, y, D)\right] .
\end{aligned}
$$

Here, similar to Eqs. (50) and (51), we have omitted the first contribution to Eq. (12) leading to an additional exponentially small factor.

The quantities $X_{00,0}$ and $X_{0}$ in Eq. (101) are defined by Eq. (13) where one should put 
$l=0$

$$
\begin{aligned}
& X_{00,0}(t, y, D)=1+\frac{1}{\tilde{v}_{F} y} \operatorname{Re} \frac{D^{2} t^{2}-\tilde{v}_{F}^{2} y^{2}}{\sqrt{\tilde{v}_{F}^{2} y^{2}-D^{2} t^{2}+D^{2}}}, \\
& X_{0}(t, y, D)=\tilde{v}_{F} y D^{2} \operatorname{Re} \frac{t^{2}-1}{\sqrt{\tilde{v}_{F}^{2} y^{2}-D^{2} t^{2}+D^{2}}} .
\end{aligned}
$$

Note that here the real part is not equal to zero only for $t \leqslant f(y, D)$, where $f(y, D)$ is defined in Eq. (52). It is easily seen that $f(y, D)<2 \mu / \Delta$ [the upper integration limit in the second contributions in Eq. (101)] if $y$ satisfies the inequality

$$
y<\frac{\sqrt{4 \mu^{2}-\Delta^{2}}}{\tilde{v}_{F} \hbar \omega_{c}} .
$$

Under the condition $\sqrt{4 \mu^{2}-\Delta^{2}}>\hbar \omega_{c}$, accepted above, this inequality is satisfied with large safety margin over the entire range of $y$ giving the major contribution to Eqs. (33) and (98). Because of this, the upper integration limits of the integrals with respect to $t$ in Eq. (101), containing the real parts indicated in Eq. (102), should be replaced with $f(y, D)$. Taking into account also that $D>1$, i.e., $D \gg \tilde{v}_{F} y$, and $t^{2}-1<\tilde{v}_{F}^{2} y^{2} / D^{2}$ over the entire domain of integration, from Eqs. (101) and (102) in the asymptotic limit $k_{B} T \ll 2 \mu-\Delta$ one obtains

$$
\begin{aligned}
& \delta_{T} \tilde{\Pi}_{00,0}(y, T, \Delta, \mu)=\frac{4 \alpha D}{\tilde{v}_{F}^{2}} \times\left[\int_{1}^{\infty} d t\left(e^{\frac{t \Delta-2 \mu}{2 k_{B} T}}+1\right)^{-1}-\int_{1}^{2 \mu / \Delta} d t\right]+\frac{4 \alpha D^{3}}{\tilde{v}_{F}^{3} y} Y(y, T, \Delta, \mu), \\
& \delta_{T} \tilde{\Pi}_{0}(y, T, \Delta, \mu)=\frac{4 \alpha \tilde{v}_{F} y^{3}}{D} Y(y, T, \Delta, \mu)
\end{aligned}
$$

where the following notation is introduced

$$
Y(y, T, \Delta, \mu) \equiv \int_{1}^{f(y, D)} d t\left[\left(e^{\frac{t \Delta-2 \mu}{2 k_{B} T}}+1\right)^{-1}-1\right] \frac{1}{\left[\tilde{v}_{F}^{2} y^{2}-D^{2}\left(t^{2}-1\right)\right]^{1 / 2}} .
$$

The first contribution to $\delta_{T} \tilde{\Pi}_{00,0}$ in Eq. (104) is easily calculated

$$
\begin{aligned}
& \frac{4 \alpha D}{\tilde{v}_{F}^{2}}\left[\int_{1}^{\infty} d t\left(e^{\frac{t \Delta-2 \mu}{2 k_{B} T}}+1\right)^{-1}-\int_{1}^{2 \mu / \Delta} d t\right]=\frac{8 \alpha}{\tilde{v}_{F}^{2} \hbar \omega_{c}}\left[k_{B} T \ln \frac{\left(1+e^{\frac{\Delta-2 \mu}{2 k_{B} T}}\right)\left(1+e^{\frac{\mu}{k_{B} T}}\right)}{\left.1+e^{-\frac{\mu}{k_{B} T}}-\mu\right]}\right. \\
& \approx \frac{8 \alpha}{\tilde{v}_{F}^{2} \hbar \omega_{c}} e^{-\frac{2 \mu-\Delta}{2 k_{B} T}} .
\end{aligned}
$$

The low-temperature behavior of the integral $Y$ defined in Eq. (105) is found in the Appendix. According to Eq. (A9) one has

$$
Y(y, T, \Delta, \mu) \approx-\frac{\tilde{v}_{F} y}{D^{2}} e^{-\frac{2 \mu-\Delta}{2 k_{B} T}} .
$$


Substituting Eqs. (106) and (107) in Eq. (104), one obtains

$$
\begin{aligned}
& \delta_{T} \tilde{\Pi}_{00,0}(y, T, \Delta, \mu) \approx \frac{4 \alpha}{\tilde{v}_{F}^{2}}\left(\frac{2 k_{B} T}{\hbar c}-D\right) e^{-\frac{2 \mu-\Delta}{2 k_{B} T}} \sim-\frac{\alpha \Delta}{\hbar \omega_{c}} e^{-\frac{2 \mu-\Delta}{2 k_{B} T}}, \\
& \delta_{T} \tilde{\Pi}_{0}(y, T, \Delta, \mu) \approx-\frac{4 \alpha \tilde{v}_{F}^{2} y^{4}}{D^{3}} e^{-\frac{2 \mu-\Delta}{2 k_{B} T}} .
\end{aligned}
$$

We note that according to Eq. (98) $\delta_{T, l=0}^{\text {expl }} \mathcal{F}_{\mathrm{TE}}$ is of the order of $\left(\delta_{T} r_{\mathrm{TE}}\right)^{2}$, i.e., $\sim\left(\delta_{T} \tilde{\Pi}_{0}\right)^{2} \sim$ $\exp \left[-2(2 \mu-\Delta) /\left(2 k_{B} T\right)\right]$ and, thus, contains an additional exponentially small factor. Because of this, we have

$$
\delta_{T, l=0}^{\operatorname{expl}} \mathcal{F}(a, T) \approx \delta_{T, l=0}^{\operatorname{expl}} \mathcal{F}_{\mathrm{TM}}(a, T) .
$$

Substituting Eqs. (85), (99), and the first equality in Eq. (108) in the TM term of Eq. (33) with $l=0$, one finally finds

$$
\delta_{T, l=0}^{\operatorname{expl}} \mathcal{F}_{\mathrm{TM}}(a, T) \sim \frac{k_{B} T Q_{0} \alpha \Delta}{a^{2} \hbar \omega_{c}} e^{-\frac{2 \mu-\Delta}{2 k_{B} T}} \int_{0}^{\infty} y^{2} d y \frac{e^{-y}}{\left(Q_{0}+2 y\right)^{3}-Q_{0}\left(Q_{0}+2 y\right) e^{-y}}
$$

Taking into account Eq. (109), the convergence of the integral which is of the order of $Q_{0}^{-3}$, and substituting the definition of $Q_{0}$ given in Eq. (83), the up to an order of magnitude behavior of $\delta_{T, l=0}^{\operatorname{expl}} \mathcal{F}$ at low temperature is

$$
\delta_{T, l=0}^{\operatorname{expl}} \mathcal{F}(a, T) \sim \frac{k_{B} T \hbar c \Delta}{\alpha a^{3} \mu^{2}} e^{-\frac{2 \mu-\Delta}{2 k_{B} T}} .
$$

We recall that this asymptotic behavior is derived under the conditions $D>1$, i.e., $\Delta>\hbar \omega_{c}$ and $\sqrt{4 \mu^{2}-\Delta^{2}}>\hbar \omega_{c}$ which are satisfied at sufficiently large separations between graphene sheets with nonzero $\Delta$ and $\mu$.

It only remains to find the low-temperature behavior of the last contribution to the thermal correction $\delta_{T, l \geqslant 1}^{\operatorname{expl}} \mathcal{F}$. We note that for $l \geqslant 1$ both the quantities $\tilde{\Pi}_{00, l}(y, 0, \Delta, \mu) \neq 0$ and $\tilde{\Pi}_{l}(y, 0, \Delta, \mu) \neq 0$ so that $\delta_{T, l \geqslant 1}^{\operatorname{expl}} \mathcal{F}$ is given by sum of all terms with $l \geqslant 1$ in Eq. (33). In doing so, it will suffice to preserve the dependence on $\tau\left(\zeta_{l}=\tau l\right)$ only in the lower integration limits of all integrals in Eq. (33) and substitute the integrands in the lowest perturbation order in $\tau$. For the TM mode, this means that one should use in Eq. (33) the second line in Eq. (85), Eq. (99), and the first line in Eq. (108). For the TE mode, according to Eq. (91), $r_{\mathrm{TE}}(0, y, 0)=0$. Because of this, $r_{\mathrm{TE}}\left(\mathrm{i} \zeta_{l}, y, 0\right)$ should be taken in the first perturbation order in $\tau$ as given by Eq. (91), whereas the thermal correction to the TE reflection coefficient is given by Eq. (100) and by the second line in Eq. (108). 
As a result, for the contribution of the TM mode one obtains

$$
\delta_{T, l \geqslant 1}^{\operatorname{expl}} \mathcal{F}_{\mathrm{TM}}(a, T) \sim \frac{k_{B} T \hbar c \Delta}{\alpha \mu^{2} a^{3}} e^{-\frac{2 \mu-\Delta}{2 k_{B} T}} \sum_{l=1}^{\infty} \int_{\zeta_{l}}^{\infty} \frac{y^{2} d y}{e^{y}-1}
$$

where we have used that $y$ giving the major contribution to the integral satisfies the condition $y \ll Q_{0}$.

For the sum of integrals in Eq. (112) we have

$$
\begin{aligned}
& \sum_{l=1}^{\infty} \int_{\zeta_{l}}^{\infty} \frac{y^{2} d y}{e^{y}-1}=\sum_{n=1}^{\infty} \frac{1}{n^{3}} \int_{n \zeta_{l}}^{\infty} d x x^{2} e^{-x}=\sum_{n=1}^{\infty}\left[\frac{2}{n^{3}} \frac{1}{e^{\tau n}-1}+\frac{2 \tau}{n^{2}} \frac{e^{\tau n}}{\left(e^{\tau n}-1\right)^{2}}+\frac{\tau^{2}}{n} \frac{e^{\tau n}\left(1+e^{\tau n}\right)}{\left(e^{\tau n}-1\right)^{3}}\right] \\
& \sim \frac{1}{\tau} \sum_{n=1}^{\infty}\left[\frac{2}{n^{4}}+\frac{2}{n^{4}}+\frac{1}{n^{4}}\right] \sim \frac{1}{\tau} .
\end{aligned}
$$

Substituting this to Eq. (112), we arrive at

$$
\delta_{T, l \geqslant 1}^{\operatorname{expl}} \mathcal{F}_{\mathrm{TM}}(a, T) \sim \frac{(\hbar c)^{2} \Delta}{\alpha \mu^{2} a^{4}} e^{-\frac{2 \mu-\Delta}{2 k_{B} T}} .
$$

The contribution of the TE mode is obtained by substituting Eqs. (91), (100), and (108) in Eq. (33) at low $T$

$$
\delta_{T, l \geqslant 1}^{\operatorname{expl}} \mathcal{F}_{\mathrm{TE}}(a, T) \sim \frac{\alpha k_{B} T}{a^{2}} \frac{Q_{2}}{D^{3}} e^{-\frac{2 \mu-\Delta}{2 k_{B} T}} \tau \sum_{l=1}^{\infty} l \int_{\zeta_{l}}^{\infty} d y e^{-y} \sim \frac{\alpha^{2} \sqrt{4 \mu^{2}-\Delta^{2}}(\hbar c)^{3}}{\Delta^{3} a^{5}} e^{-\frac{2 \mu-\Delta}{2 k_{B} T}}
$$

It is easily seen that the quantities in Eqs. (114) and (115) can be of the same order of magnitude. Thus, for the total contribution $\delta_{T, l \geqslant 1}^{\operatorname{expl}} \mathcal{F}$ we obtain

$$
\delta_{T, l \geqslant 1}^{\operatorname{expl}} \mathcal{F}(a, T) \sim \frac{(\hbar c)^{2}}{a^{4}}\left(\frac{\Delta}{\alpha \mu^{2}}+\frac{\alpha^{2} \hbar c \sqrt{4 \mu^{2}-\Delta^{2}}}{a \Delta^{3}}\right) e^{-\frac{2 \mu-\Delta}{2 k_{B} T}}
$$

This result is derived for $\mu>0$ and $\Delta>0$.

From Eqs. (97), (111), and (116), one concludes that the main term in the lowtemperature behavior of the Casimir free energy for graphene with $\Delta<2 \mu$ is determined by the TE mode in the implicit contribution given by Eq. (97). Substituting Eq. (97) in Eq. (72) one arrives at the Casimir entropy at low temperature

$$
S(a, T) \sim \frac{\alpha^{2} a\left(4 \mu^{2}-\Delta^{2}\right) k_{B}^{2} T}{(\hbar c)^{3}} .
$$

In the limit of vanishing temperature, the Casimir entropy (117) goes to zero in agreement with the Nernst heat theorem. 
The results of this section were derived under the conditions

$$
k_{B} T \ll \frac{\hbar v_{F}}{2 a} \ll \frac{\hbar c}{2 a}<\Delta, \quad k_{B} T \ll 2 \mu-\Delta .
$$

Thus, although the first two expansion parameters in Eq. (75) remain the same, the third one is replaced with

$$
e^{-\frac{2 \mu-\Delta}{2 k_{B} T}} \ll 1
$$

One more condition used in the derivation of expressions (82) for the polarization tensor is

$$
\frac{\hbar c}{2 a}<\sqrt{4 \mu^{2}-\Delta^{2}} .
$$

These application conditions are discussed in Sec. VII.

\section{CONCLUSIONS AND DISCUSSION}

In this paper, we have found the low-temperature behavior of the Casimir free energy and entropy of two real graphene sheets possessing the nonzero energy gap and chemical potential. This problem is solved analytically in the framework of the Dirac model. The response of graphene to the electromagnetic field is described on the basis of first principles of thermal quantum field theory by means of the polarization tensor in $(2+1)$-dimensional space-time. The thermal correction to the Casimir energy of two parallel graphene sheets at zero temperature is presented as a sum of two contributions. The first of them, called implicit, contains the polarization tensor at zero temperature, and the dependence of this contribution on temperature is determined by a summation over the Matsubara frequencies. The temperature dependence of the second contribution, called explicit, is determined by the thermal correction to the polarization tensor. The low-temperature behaviors of both contributions were found for different relationships between the energy gap and chemical potential of graphene sheets, i.e., for $\Delta>2 \mu, \Delta=2 \mu$, and $\Delta<2 \mu$, and turned out to be essentially different.

According to the results of Sec. IV, which are repeated here by presenting only the dimensional quantities, the low-temperature behavior of the Casimir free energy and entropy for graphene sheets with $\Delta>2 \mu$ is eventually determined by the TE mode in an implicit contribution to the thermal correction

$$
\delta_{T} \mathcal{F}(a, T) \sim-\frac{\left(k_{B} T\right)^{5}}{(\hbar c)^{2} \Delta^{2}}, \quad S(a, T) \sim \frac{k_{B}^{5} T^{4}}{(\hbar c)^{2} \Delta^{2}},
$$


and it does not depend on the chemical potential.

In Sec. $\mathrm{V}$ it is shown that for graphene sheets with $\Delta=2 \mu$ the eventual low-temperature behavior of the Casimir free energy and entropy is determined by the TM mode in an explicit contribution to the thermal correction

$$
\delta_{T} \mathcal{F}(a, T) \sim-\frac{k_{B} T}{a^{2}}, \quad S(a, T) \sim \frac{k_{B}}{a^{2}}
$$

Finally, as shown in Sec. VI, for the case $\Delta<2 \mu$ the low-temperature behavior of the Casimir free energy and entropy is governed by the TE mode in an implicit contribution to the thermal correction given by

$$
\delta_{T} \mathcal{F}(a, T) \sim-\frac{a\left(4 \mu^{2}-\Delta^{2}\right)\left(k_{B} T\right)^{2}}{(\hbar c)^{3}}, \quad S(a, T) \sim \frac{a\left(4 \mu^{2}-\Delta^{2}\right) k_{B}^{2} T}{(\hbar c)^{3}} .
$$

It is interesting to compare these results with the case of a pristine graphene with $\Delta=$ $\mu=0$ where [76]

$$
\delta_{T} \mathcal{F}(a, T) \sim \frac{\left(k_{B} T\right)^{3}}{(\hbar c)^{2}} \ln \frac{a k_{B} T}{\hbar c}, \quad S(a, T) \sim-k_{B} \frac{\left(k_{B} T\right)^{2}}{(\hbar c)^{2}} \ln \frac{a k_{B} T}{\hbar c} .
$$

As is seen from the comparison of Eqs. (121)-(123) with Eq. (124), for real graphene sheets there is a nontrivial interplay between the values of $\Delta$ and $\mu$ which leads to different behaviors of the Casimir energy and entropy with vanishing temperature, especially in the case $\Delta<2 \mu$ where the polarization tensor at $T=0$ depends on $\mu$.

From Eqs. (121) and (123) one concludes that the Casimir entropy is positive and vanishes with vanishing temperature, i.e., for graphene with $\Delta>2 \mu$ and $\Delta<2 \mu$ the Nernst heat theorem is satisfied and, thus, the Lifshitz theory of the Casimir interaction is consistent with the requirements of thermodynamics (the same holds for a pristine graphene). According to Eq. (122), this is, however, not so for graphene with $\Delta=2 \mu \neq 0$ where the Casimir entropy at zero temperature is not equal to zero and its value depends on the parameter of a system (volume). As discussed in Sec. V, however, this anomaly is not relevant to any physical situation because for real graphene samples the exact equality $\Delta=2 \mu$ is not realizable. We note that the real part of the electrical conductivity of graphene as a function of frequency also experiences a qualitative change when the energy gap $\Delta$ decreases from $\Delta>2 \mu$ to $\Delta<2 \mu[60]$.

It should be noted that the asymptotic expressions (121) and (123) are not applicable to graphene sheets with too small values of $\Delta-2 \mu$ and $2 \mu-\Delta$, respectively. The point is that 
if the values of $\Delta$ and $2 \mu$ are too close to each other the exponentially small parameters in Eqs. (75) and (119) lose their meaning and cannot be used. Taking into account that the polarization tensor is a continuous function of $\Delta$ at the point $\Delta=2 \mu$, the possibility exists that an apparent discontinuity of the obtained asymptotic formulas at $\Delta=2 \mu$ may be an artifact of the expansion in small parameters at the crossover region. For a comprehensive resolution of this question, it would be desirable to find the more exact asymptotic expressions applicable for the values of $2 \mu$ arbitrarily close to $\Delta$ from the left and from the right. In future it is also interesting to investigate the case of two dissimilar graphene sheets with different values of the energy gap and chemical potential. The configuration of a graphene sheet interacting with an ideal metal plane (it has been known that for two ideal metal planes the Casimir entropy satisfies the Nernst heat theorem [87]) or a plate made of conventional metallic or dielectric materials.

According to Sec. I, theoretical description of the Lifshitz theory using the polarization tensor of graphene [75] is in good agreement with the experiment on measuring the Casimir interaction in graphene system [74]. Taking into consideration that the polarization tensor of graphene results in two spatially nonlocal, complex dielectric permittivities (the longitudinal one and the transverse one [50]), it may be suggested that a more fundamental theoretical description of the dielectric response of metals admits a similar approach. In application to metals, the nonlocal dielectric permittivities of this kind could lead to almost the same results, as the dissipative Drude model, for the propagating waves on the mass shell, but deviate from them significantly for the evanescent fields off the mass shell (in contrast to the nonlocal dielectric functions describing the anomalous skin effect [88]). In such a manner graphene might point the way for resolution of the Casimir puzzle which remains unresolved for already 20 years.

\section{Acknowledgments}

The work of G.L.K. and V.M.M. was partially supported by the Peter the Great Saint Petersburg Polytechnic University in the framework of the Program "5-100-2020". The work of V.M.M. was partially funded by the Russian Foundation for Basic Research, Grant No. 19-02-00453 A. His work was also partially supported by the Russian Government Program of Competitive Growth of Kazan Federal University. 


\section{Appendix A}

Here, we derive the low-temperature behavior of two integrals used in the main text. We begin with the integral $I_{00,0}^{(2)}$ defined in Eqs. (51) and (52). To calculate the quantity $I_{00,0}^{(2)}$ in the case $\Delta>2 \mu$ we introduce the integration variable $v=t-1$ and obtain

$$
I_{00,0}^{(2)} \approx e^{-\frac{\Delta-2 \mu}{2 k_{B} T}} \int_{0}^{f(y, D)-1} d v e^{-v \frac{\Delta}{2 k_{B} T}} \frac{D^{2}(v+1)^{2}}{\left[\tilde{v}_{F}^{2} y^{2}-D^{2} v(v+2)\right]^{1 / 2}},
$$

where we have omitted the negligibly small quantity $\tilde{v}_{F}^{2} y^{2}$ taking into account that the dominant contribution to Eq. (33) is given by $y \sim 1$. Under this condition $f(1, D)-1 \ll 1$ and, thus, $v \ll 1$. Then the asymptotic behavior of Eq. (A1) at low $T$ can be estimated as

$$
\begin{gathered}
I_{00,0}^{(2)} \sim e^{-\frac{\Delta-2 \mu}{2 k_{B} T}} \int_{0}^{f(1, D)-1} d v e^{-v \frac{\Delta}{2 k_{B} T}} \frac{D^{2}}{\left(\tilde{v}_{F}^{2}-2 D^{2} v\right)^{1 / 2}} \\
=D \frac{2 k_{B} T}{\Delta} e^{-\frac{\Delta-2 \mu}{2 k_{B} T}} \int_{0}^{U(D, T)} d u \frac{e^{-u}}{\left(\frac{\tilde{v}_{F}^{2}}{D^{2}}-4 \frac{k_{B} T}{\Delta} u\right)^{1 / 2}},
\end{gathered}
$$

where $u=v \Delta /\left(2 k_{B} T\right)$ is the integration variable introduced in place of $v$, and $U(D, T) \equiv$ $\Delta[f(1, D)-1] /\left(2 k_{B} T\right)$. In view of the fact that $4 k_{B} T u / \Delta$ goes to zero when $T$ vanishes and the main contribution to the integral is given by $u \sim 1$, we find

$$
I_{00,0}^{(2)} \sim \frac{D^{2}}{\tilde{v}_{F}} \frac{k_{B} T}{\Delta} e^{-\frac{\Delta-2 \mu}{2 k_{B} T}} \int_{0}^{\infty} d u e^{-u}=\frac{k_{B} T}{\tilde{v}_{F}} \frac{\Delta}{\left(\hbar \omega_{c}\right)^{2}} e^{-\frac{\Delta-2 \mu}{2 k_{B} T}} .
$$

Now we consider the same integral but for graphene with $\Delta=2 \mu$. For this purpose, we again begin from Eq. (51), where now $\Delta=2 \mu$, and substitute there the identity

$$
\left[e^{\frac{(t-1) \Delta}{2 k_{B} T}}+1\right]^{-1}=\sum_{n=1}^{\infty}(-1)^{n-1} e^{-n(t-1) \frac{\Delta}{2 k_{B} T}} .
$$

Then, after introducing the integration variable $v=t-1$, one obtains instead of Eq. (A2)

$$
\begin{aligned}
& I_{00,0}^{(2)} \sim \sum_{n=1}^{\infty}(-1)^{n-1} \int_{0}^{f(1, D)-1} d v e^{-n v \frac{\Delta}{2 k_{B} T}} \frac{D^{2}}{\left(\tilde{v}_{F}^{2}-2 D^{2} v\right)^{1 / 2}} \\
& =D \frac{2 k_{B} T}{\Delta} \sum_{n=1}^{\infty} \frac{(-1)^{n-1}}{n} \int_{0}^{n U(D, T)} d u \frac{e^{-u}}{\left(\frac{\tilde{v}_{F}^{2}}{D^{2}}-4 \frac{k_{B} T}{n \Delta} u\right)^{1 / 2}}
\end{aligned}
$$

where $u=n v \Delta /\left(2 k_{B} T\right)$. For arbitrarily small $T$ this equation can be rearranged as

$$
I_{00,0}^{(2)} \sim \frac{D^{2}}{\tilde{v}_{F}} \frac{k_{B} T}{\Delta} \sum_{n=1}^{\infty} \frac{(-1)^{n-1}}{n} \int_{0}^{\infty} d u e^{-u}=\frac{k_{B} T}{\tilde{v}_{F}} \frac{\Delta}{\left(\hbar \omega_{c}\right)^{2}} \ln 2 .
$$


Now we find the low-temperature behavior of the integral $Y$ defined in Eq. (105). The power of exponent in Eq. (105) is negative over the entire integration range. Because of this, one can use the following expansion

$$
\begin{aligned}
& Y(y, T, \Delta, \mu)=\int_{1}^{f(y, D)} d t\left[\sum_{n=1}^{\infty}(-1)^{n-1} e^{\frac{t \Delta-2 \mu}{2 k_{B} T}(n-1)}-1\right] \frac{1}{\left[\tilde{v}_{F}^{2} y^{2}-D^{2}\left(t^{2}-1\right)\right]^{1 / 2}} \\
& \quad=-\sum_{k=1}^{\infty}(-1)^{k-1} \int_{1}^{f(y, D)} d t e^{\frac{t \Delta-2 \mu}{2 k_{B} T} k} \frac{1}{\left[\tilde{v}_{F}^{2} y^{2}-D^{2}\left(t^{2}-1\right)\right]^{1 / 2}} .
\end{aligned}
$$

Now we replace the integration variable $t$ with $v=t-1$ and take into account that for $y \sim 1$

$$
f(y, D)-1 \approx \frac{\tilde{v}_{F}^{2} y^{2}}{2 D^{2}} \ll 1 .
$$

For this reason, one can neglect by $v$ as compared to unity in the power of exponent and also in the denominator of Eq. (A7). In the sum, we can restrict ourselves by only the first term because all other terms contain additional exponentially small factors as compared with it. The result is

$$
Y(y, T, \Delta, \mu) \approx-e^{-\frac{2 \mu-\Delta}{2 k_{B} T}} \int_{0}^{f(y, D)-1} \frac{d v}{\left(\tilde{v}_{F}^{2} y^{2}-2 D^{2} v\right)^{1 / 2}} \approx-\frac{\tilde{v}_{F} y}{D^{2}} e^{-\frac{2 \mu-\Delta}{2 k_{B} T}},
$$

where we have used the condition (A8).

[1] H. B. G. Casimir, On the attraction between two perfectly conducting plates, Proc. Kon. Ned. Akad. Wet. B 51, 793 (1948).

[2] M. Bordag, G. L. Klimchitskaya, U. Mohideen, and V. M. Mostepanenko, Advances in the Casimir Effect (Oxford University Press, Oxford, 2015).

[3] T. Emig, N. Graham, R. L. Jaffe, and M. Kardar, Casimir forces between compact objects: The scalar case, Phys. Rev. D 77, 025005 (2008).

[4] S. J. Rahi, T. Emig, N. Graham, R. L. Jaffe, and M. Kardar, Scattering theory approach to electromagnetic Casimir forces, Phys. Rev. D 80, 085021 (2009).

[5] R. Passante, L. Rizzuto, S. Spagnolo, S. Tanaka, and T. Y. Petrosky, Harmonic oscillator model for the atom-surface Casimir-Polder interaction energy, Phys. Rev. A 85, 062109 (2012).

[6] M. I. Katsnelson, Graphene: Carbon in Two Dimensions (Cambridge University Press, Cambridge, 2012). 
[7] A. H. Castro Neto, F. Guinea, N. M. R. Peres, K. S. Novoselov, and A. K. Geim, The electronic properties of graphene, Rev. Mod. Phys. 81, 109 (2009).

[8] S. P. Gavrilov and D. M. Gitman, Vacuum instability in external fields, Phys. Rev. D 53, 7162 (1996).

[9] D. M. Gitman and S. I. Zlatev, Spin factor in the path integral representation of the Dirac propagator in external fields, Phys. Rev. D 55, 7701 (1997).

[10] V. P. Gusynin and I. A. Shovkovy, Derivative expansion of the effective action for quantum electrodynamics in $2+1$ and $3+1$ dimensions, J. Math. Phys. 40, 5406 (1999).

[11] Qiong-gui Lin, Electron-positron pair creation in a vacuum by an electromagnetic field in $3+1$ and lower dimensions, J. Phys. G: Nucl. Part. Phys. 25, 17 (1999).

[12] M. I. Katsnelson, K. S. Novoselov and A. K. Geim, Chiral tunnelling and the Klein Paradox in graphene, Nat. Phys. 2, 620 (2006).

[13] D. Allor, T. D. Cohen, and D. A. McGady, Schwinger mechanism and graphene, Phys. Rev. D 78, 096009 (2008).

[14] C. G. Beneventano, P. Giacconi, E. M. Santangelo, and R. Soldati, Planar QED at finite temperature and density: Hall conductivity, Berry's phases and minimal conductivity of graphene, J. Phys. A 42, 275401 (2009).

[15] S. P. Gavrilov, D. M. Gitman, and N. Yokomizo, Dirac fermions in strong electric field and quantum transport in graphene, Phys. Rev. D 86, 125022 (2012).

[16] G. L. Klimchitskaya and V. M. Mostepanenko, Creation of quasiparticles in graphene by a time-dependent electric field, Phys. Rev. D 87, 125011 (2013).

[17] I. Akal, R. Egger, C. Müller, and S. Villarba-Chávez, Low-dimensional approach to pair production in an oscillating electric field: Application to bandgap graphene layers, Phys. Rev. D 93, 116006 (2016).

[18] A. Golub, C. Müller, and S. Villarba-Chávez, Dimensionality-Driven Photo Production of Massive Dirac Pairs Near Threshold in Gapped Graphene Monolayers, Phys. Rev. Lett. 124, $110403(2020)$.

[19] M. Dressel and G. Grüner, Electrodynamics of Solids: Optical Properties of Electrons in Metals (Cambridge University Press, Cambridge, 2003).

[20] J. F. Dobson, A. White, and A. Rubio, Asymptotics of the dispersion interaction: Analytic benchmarks for van der Waals energy functionals, Phys. Rev. Lett. 96, 073201 (2006). 
[21] G. Gómez-Santos, Thermal van der Waals interaction between graphene layers, Phys. Rev. B 80, 245424 (2009).

[22] D. Drosdoff and L. M. Woods, Casimir forces and graphene sheets, Phys. Rev. B 82, 155459 (2010).

[23] D. Drosdoff and L. M. Woods, Casimir interaction between graphene sheets and metamaterials, Phys. Rev. A 84, 062501 (2011).

[24] Bo E. Sernelius, Casimir interactions in graphene systems, Europhys. Lett. 95, 57003 (2011).

[25] T. E. Judd, R. G. Scott, A. M. Martin, B. Kaczmarek, and T. M. Fromhold, Quantum reflection of ultracold atoms from thin films, graphene and semiconductor heterostructures, New J. Phys. 13, 083020 (2011).

[26] J. Sarabadani, A. Naji, R. Asgari, and R. Podgornik, Many-body effects in the van der WaalsCasimir interaction between graphene layers, Phys. Rev. B 84, 155407 (2011); Phys. Rev. B 87, 239905(E) (2013).

[27] D. Drosdoff, A. D. Phan, L. M. Woods, I. V. Bondarev, and J. F. Dobson, Effects of spatial dispersion on the Casimir force between graphene sheets, Eur. Phys. J. B 85, 365 (2012).

[28] Bo E. Sernelius, Retarded interactions in graphene systems, Phys. Rev. B 85, 195427 (2012).

[29] W.-K. Tse and A. H. MacDonald, Quantized Casimir Force, Phys. Rev. Lett. 109, 236806 (2012).

[30] A. D. Phan, L. M. Woods, D. Drosdoff, I. V. Bondarev, and N. A. Viet, Temperature dependent graphene suspension due to thermal Casimir interaction, Appl. Phys. Lett. 101, 113118 (2012).

[31] A. D. Phan, N. A. Viet, N. A. Poklonski, L. M. Woods, and C. H. Le, Interaction of a graphene sheet with a ferromagnetic metal plate, Phys. Rev. B 86, 155419 (2012).

[32] S. Ribeiro and S. Scheel, Shielding vacuum fluctuations with graphene, Phys. Rev. A 88, 042519 (2013); 89 039904(E) (2014).

[33] T. Cysne, W. J. M. Kort-Kamp, D. Oliver, F. A. Pinheiro, F. S. S. Rosa, and C. Farina, Tuning the Casimir-Polder interaction via magneto-optical effects in graphene, Phys. Rev. A 90, 052511 (2014).

[34] N. Knusnutdinov, R. Kashapov, and L. M. Woods, Casimir-Polder effect for a stack of conductive planes, Phys. Rev. A 94, 012513 (2016).

[35] N. Inui, Casimir effect on graphene resonator, J. Appl. Phys. 119, 104502 (2016). 
[36] N. Knusnutdinov, R. Kashapov, and L. M. Woods, Thermal Casimir and Casimir-Polder interactions in N parallel 2D Dirac materials, 2D Materials 5, 035032 (2018).

[37] A. Derras-Chouk, E. M. Chudnovsky, D. A. Garanin, and R. Jaafar, Graphene cantilever under Casimir force, J. Phys. D: Appl. Phys. 51, 195301 (2018).

[38] P. K. Pyatkovsky, Dynamical polarization, screening, and plasmons in gapped graphene, J. Phys.: Condens. Matter 21, 025506 (2009).

[39] M. Bordag, I. V. Fialkovsky, D. M. Gitman, and D. V. Vassilevich, Casimir interaction between a perfect conductor and graphene described by the Dirac model, Phys. Rev. B 80, 245406 (2009).

[40] I. V. Fialkovsky, V. N. Marachevsky, and D. V. Vassilevich, Finite-temperature Casimir effect for graphene, Phys. Rev. B 84, 035446 (2011).

[41] M. Bordag, G. L. Klimchitskaya, and V. M. Mostepanenko, Thermal Casimir effect in the interaction of graphene with dielectrics and metals, Phys. Rev. B 86, 165429 (2012).

[42] M. Chaichian, G. L. Klimchitskaya, V. M. Mostepanenko, and A. Tureanu, Thermal CasimirPolder interaction of different atoms with graphene, Phys. Rev. A 86, 012515 (2012).

[43] G. L. Klimchitskaya and V. M. Mostepanenko, Van der Waals and Casimir interactions between two graphene sheets, Phys. Rev. B 87, 075439 (2013).

[44] B. Arora, H. Kaur, and B. K. Sahoo, $C_{3}$ coefficients for the alkali atoms interacting with a graphene and carbon nanotube, J. Phys. B 47, 155002 (2014).

[45] K. Kaur, J. Kaur, B. Arora, and B. K. Sahoo, Emending thermal dispersion interaction of Li, $\mathrm{Na}, \mathrm{K}$ and Rb alkali-metal atoms with graphene in the Dirac model, Phys. Rev. B 90, 245405 (2014).

[46] G. L. Klimchitskaya and V. M. Mostepanenko, Classical Casimir-Polder force between polarizable microparticles and thin films including graphene, Phys. Rev. A 89, 012516 (2014).

[47] G. L. Klimchitskaya and V. M. Mostepanenko, Classical limit of the Casimir interaction for thin films with applications to graphene, Phys. Rev. B 89, 035407 (2014).

[48] G. L. Klimchitskaya and V. M. Mostepanenko, Observability of thermal effects in the Casimir interaction from graphene-coated substrates, Phys. Rev. A 89, 052512 (2014).

[49] G. L. Klimchitskaya and V. M. Mostepanenko, Impact of graphene coating on the atom-plate interaction, Phys. Rev. A 89, 062508 (2014).

[50] G. L. Klimchitskaya, V. M. Mostepanenko, and Bo E. Sernelius, Two approaches for describing 
the Casimir interaction with graphene: density-density correlation function versus polarization tensor, Phys. Rev. B 89, 125407 (2014).

[51] M. Bordag, G. L. Klimchitskaya, V. M. Mostepanenko, and V. M. Petrov, Quantum field theoretical description for the reflectivity of graphene, Phys. Rev. D 91, 045037 (2015); 93, 089907(E) (2016).

[52] M. Bordag, I. Fialkovskiy, and D. Vassilevich, Enhanced Casimir effect for doped graphene, Phys. Rev. B 93, 075414 (2016); 95, 119905(E) (2017).

[53] G. L. Klimchitskaya and V. M. Mostepanenko, Origin of large thermal effect in the Casimir interaction between two graphene sheets, Phys. Rev. B 91, 174501 (2015).

[54] G. L. Klimchitskaya, Quantum field theory of the Casimir force for graphene, Int. J. Mod. Phys. A 31, 1641026 (2016).

[55] G. Bimonte, G. L. Klimchitskaya, and V. M. Mostepanenko, How to observe the giant thermal effect in the Casimir force for graphene systems, Phys. Rev. A 96, 012517 (2017).

[56] G. Bimonte, G. L. Klimchitskaya, and V. M. Mostepanenko, Thermal effect in the Casimir force for graphene and graphene-coated substrates: Impact of nonzero mass gap and chemical potential, Phys. Rev. B 96, 115430 (2017).

[57] C. Henkel, G. L. Klimchitskaya, and V. M. Mostepanenko, Influence of chemical potential on the Casimir-Polder interaction between an atom and gapped graphene or graphene-coated substrate, Phys. Rev. A 97, 032504 (2018).

[58] G. L. Klimchitskaya, V. M. Mostepanenko, and V. M. Petrov, Impact of chemical potential on the reflectance of graphene in the infrared and microwave domains, Phys. Rev. A 98, 023809 (2018).

[59] G. L. Klimchitskaya, V. S. Malyi, V. M. Mostepanenko, and V. M. Petrov, Reflectance of graphene-coated dielectric plates in the framework of Dirac model: joint action of energy gap and chemical potential, J. Phys.: Condens. Matter 31, 505003 (2019).

[60] G. L. Klimchitskaya, V. M. Mostepanenko, and V. M. Petrov, Conductivity of graphene in the framework of Dirac model: Interplay between nonzero mass gap and chemical potential, Phys. Rev. B 96, 235432 (2017).

[61] G. L. Klimchitskaya and V. M. Mostepanenko, Kramers-Kronig relations and causality conditions for graphene in the framework of Dirac model, Phys. Rev. D 97085001 (2018).

[62] G. L. Klimchitskaya, U. Mohideen, and V. M. Mostepanenko, The Casimir force between real 
materials: Experiment and theory, Rev. Mod. Phys. 81, 1827 (2009).

[63] L. M. Woods, D. A. R. Dalvit, A. Tkatchenko, P. Rodriguez-Lopez, A. W. Rodriguez, and R. Podgornik, Materials perspective on Casimir and van der Waals interactions, Rev. Mod. Phys. 88, 045003 (2016).

[64] G. Bimonte, D. López, and R. S. Decca, Isoelectronic determination of the thermal Casimir force, Phys. Rev. B 93, 184434 (2016).

[65] J. Xu, G. L. Klimchitskaya, V. M. Mostepanenko, and U. Mohideen, Reducing detrimental electrostatic effects in Casimir-force measurements and Casimir-force-based microdevices, Phys. Rev. A 97, 032501 (2018).

[66] M. Liu, J. Xu, G. L. Klimchitskaya, V. M. Mostepanenko, and U. Mohideen, Examining the Casimir puzzle with an upgraded AFM-based technique and advanced surface cleaning, Phys. Rev. B 100, 081406(R) (2019).

[67] M. Liu, J. Xu, G. L. Klimchitskaya, V. M. Mostepanenko, and U. Mohideen, Precision measurements of the gradient of the Casimir force between ultraclean metallic surfaces at larger separations, Phys. Rev. A 100, 052511 (2019).

[68] M. Bordag, Low Temperature Expansion in the Lifshitz Formula, Adv. Math. Phys. 2014, $981586(2014)$.

[69] G. L. Klimchitskaya and C. C. Korikov, Analytic results for the Casimir free energy between ferromagnetic metals, Phys. Rev. A 91, 032119 (2015).

[70] G. L. Klimchitskaya and C. C. Korikov, Casimir entropy for magnetodielectrics, J. Phys.: Condens. Matter 27, 214007 (2015).

[71] G. L. Klimchitskaya and V. M. Mostepanenko, Low-temperature behavior of the Casimir free energy and entropy of metallic films, Phys. Rev. A 95, 012130 (2017).

[72] G. L. Klimchitskaya and V. M. Mostepanenko, Casimir free energy of dielectric films: classical limit, low-temperature behavior and control, J. Phys.: Condens. Matter 29, 275701 (2017).

[73] C. C. Korikov and V. M. Mostepanenko, Nernst heat theorem for the Casimir-Polder interaction between a magnetizable atom and ferromagnetic dielectric plate, Mod. Phys. Lett. A 35, $2040010(2020)$.

[74] A. A. Banishev, H. Wen, J. Xu, R. K. Kawakami, G. L. Klimchitskaya, V. M. Mostepanenko, and U. Mohideen, Measuring the Casimir force gradient from graphene on a $\mathrm{SiO}_{2}$ substrate Phys. Rev. B 87, 205433 (2013). 
[75] G. L. Klimchitskaya, U. Mohideen, and V. M. Mostepanenko, Theory of the Casimir interaction for graphene-coated substrates using the polarization tensor and comparison with experiment, Phys. Rev. B 89, 115419 (2014).

[76] V. B. Bezerra, G. L. Klimchitskaya, V. M. Mostepanenko, and C. Romero, Nernst heat theorem for the thermal Casimir interaction between two graphene sheets, Phys. Rev. A 94, 042501 (2016).

[77] G. L. Klimchitskaya and V. M. Mostepanenko, Low-temperature behavior of the CasimirPolder free energy and entropy for an atom interacting with graphene, Phys. Rev. A 98, 032506 (2018).

[78] G. L. Klimchitskaya, The Casimir-Polder interaction of an atom and real graphene sheet: Verification of the Nernst heat theorem, Mod. Phys. Lett. A 35, 2040004 (2020).

[79] G. L. Klimchitskaya and V. M. Mostepanenko, The Nernst heat theorem for an atom interacting with graphene: Dirac model with nonzero energy gap and chemical potential, Phys. Rev. D 101, 116003 (2020).

[80] N. Khusnutdinov and N. Emelianova, Low-temperature expansion of the Casimir-Polder free energy for an atom interacting with a conducting plane, Int. J. Mod. Phys. A 34, 1950008 (2019).

[81] V. P. Gusynin, S. G. Sharapov, and J. P. Carbotte, On the universal ac optical background in graphene, New J. Phys. 11, 095013 (2009)

[82] L. A. Falkovsky, Optical properties of graphene, J. Phys.: Conf. Series 129, 012004 (2008).

[83] E. M. Lifshitz and L. P. Pitaevskii, Statistical Physics, Part II (Pergamon, Oxford, 1980).

[84] A. Erdélyi, W. Magnus, F. Oberhettinger, and F. G. Tricomi, Higher Transcendental Functions, Vol. 1 (Kriger, New York, 1981).

[85] L. D. Landau and E. M. Lifshitz, Statistical Physics, Part I (Pergamon, Oxford, 1980).

[86] Yu. B. Rumer and M. S. Ryvkin, Thermodynamics, Statistical Physics, and Kinetics (Mir, Moscow, 1980).

[87] H. Mitter and D. Robaschik, Thermodynamics of the Casimir effect, Eur. Phys. J. B 13, 335 (2000).

[88] K. L. Kliewer and R. Fuchs, Anomalous Skin Effect for Specular Electron Scattering and Optical Experiments at Non-Normal Angles of Incidence, Phys. Rev. 172, 607 (1968). 